\title{
Gestión de datos de investigación y bibliotecas: preservando los nuevos bienes científicos
}

\author{
Paloma Marín-Arraiza \\ Universidade Estadual Paulista, Brasil \\ paloma.arraiza@unesp.br \\ https://orcid.org/0000-0001-7460-7794
}

\author{
Mirelys Puerta-Díaz \\ Universidade Estadual Paulista, Brasil \\ mirelys.puerta@unesp.br \\ https://orcid.org/0000-0002-2312-2540
}

\author{
Silvana Gregorio-Vidotti \\ Universidade Estadual Paulista, Brasil \\ silvana.vidotti@unesp.br \\ https://orcid.org/0000-0002-4216-0374
}

\section{Research data management and libraries: preserving the new scientific assets}

\section{RESUMEN}

Los datos se han convertido en la base de la infraestructura de la ciencia. La implementación de políticas y servicios de gestión de datos de investigación (GDI) en contextos académicos deviene en factor crítico del éxito organizacional, lo que presenta nuevas oportunidades para el desarrollo de las bibliotecas universitarias y de investigación. A partir de un análisis bibliográfico sobre GDI y bibliotecas y otro exploratorio sobre los servicios de 34 bibliotecas universitarias y de investigación en Europa y América Latina, se extraen los principales puntos de actuación de estas en los procesos de GDI. Para completar el análisis exploratorio, se incluyen los resultados sobre los servicios de identificadores persistentes (PIDs). En muchos casos, los servicios de GDI se convierten en modelos de cooperación con otras unidades institucionales y los investigadores. Asimismo, se discuten las competencias de dos perfiles profesionales necesarios en este contexto: el bibliotecario de datos y el administrador de datos.

PALABRAS CLAVE

Gestión de datos de investigación; Servicios bibliotecarios; Bibliotecario de datos; Identificadores persistentes; Repositorio de datos

\section{ABSTRACT}

Data has become the basis of the science infrastructure. The implementation of research data management (GDI) policies and services in academic contexts becomes a critical factor of organizational success, presenting new opportunities for the development of university and research libraries. Based on a bibliographic analysis of GDI and libraries and an exploratory analysis of the services of 34 university and research libraries in Europe and Latin America, the main points of action of these in the GDI processes are extracted. The results on services of persistent identifiers (PIDs) are included to complete the exploratory analysis. In many cases, GDI services become models of cooperation with other institutional units and researchers. It also discusses the competencies of two professional profiles needed in this context: the data librarian and the data steward.

\section{KEYWORDS}

Research data management; Library services; Data librarian; Persistent identifiers; Data repository 


\section{Introducción}

El ecosistema de investigación ha cambiado sustancialmente en la última década. Los recientes avances, en las tecnologías de la información y las comunicaciones, el acelerado incremento de la producción científica global, así como la emergencia y fortalecimiento de directivas nacionales en pos del fomento de la calidad en el proceso de comunicación científica, han suscitado un creciente interés en el papel de los datos científicos en este ecosistema (Amorim, Castro, Rocha da Silva y Ribeiro, 2017; Corrall, 2014; Corrall, Kennan y Afzal, 2013; Hey, Tansley y Tolle, 2009).

La práctica científica se encuandra ahora en el denominado paradigma impulsado por datos (data-driven) o Cuarto Paradigma de la ciencia (Hey et al., 2009). En él, los datos de investigación desempeñan un papel fundamental a lo largo del proceso científico, desde la fase de planificación hasta la publicación de los resultados de investigación. Entre las razones que han guiado la compartición de los datos Maredata (2018) destaca la necesidad de la comunidad científica de maximizar la transparencia y la fiabilidad de los datos; permitir la verificación de los resultados de investigación; facilitar la colaboración entre usuarios de datos, creadores de datos y reutilizadores, entre otras.

Puesto que los datos son cruciales en procesos como los controles de reproducibilidad, innovación y toma de decisiones, representan un importante e inestimable capital intelectual dentro del ecosistema de investigación (Mons, 2018; Kowalczyk y Shankar, 2011).

Es importante destacar que el término «datos de investigación» hace referencia a todo tipo de información (sin importar su formato) necesaria para sostener y validar el proceso científico. En los datos de investigación se incluyen todos los materiales creados en el transcurso del trabajo de investigación, por ejemplo, mediante digitalizaciones, grabaciones, capturas fotográficas, experimentos, mediciones, encuestas y entrevistas. Para garantizar su reproducibilidad, los datos deben estar en conformidad con los principios FAIR (encontrable, accesible, interoperable, reutilizable, del inglés findable, accessible, interoperable, reusable). Estos principios se establecieron para proporcionar unas directrices y medidas para la correcta gestión de datos. Además, no solo se aplican a los datos, sino también a los sistemas de información (Wilkinson et al., 2016).

Si bien la responsabilidad por la gestión de datos recae en primera instancia en los investigadores, resulta necesario que las instituciones ofrezcan el apoyo necesario para adaptarse a los nuevos requisitos con respecto a la gestión de datos. Por ello, se demanda la creación de infraestructuras y servicios específicos, lo que impulsa a optimizar los procesos, procedimientos y servicios institucionales en función de esta meta (Perrier y
Barnes, 2018; Amorim et al., 2017; Higman y Pinfield, 2015).

Estos servicios de apoyo a la investigación requieren la intervención de diferentes profesionales, por lo que suelen repartirse entre diferentes unidades institucionales (bibliotecas, servicios informáticos, comités de ética, entre otros). En las bibliotecas universitarias y de investigación, la gestión de datos de investigación (GDI, del inglés RDM-Research Data Management) debe formar parte del desarrollo natural de las tareas ejercidas (Alonso-Arévalo, 2019) y verse como una oportunidad para entrar en los flujos de trabajo de los investigadores.

Al implementar nuevos servicios se persigue conseguir una mejor y más cuidadosa administración de datos (data stewardship) y apoyar directamente las tareas investigativas a lo largo de todo su ciclo. Las buenas prácticas en la gestión de datos garantizan, que más allá de los propósitos para los que fueron creados los datos originalmente, puedan ser reutilizados para el descubrimiento de nuevo conocimiento, hecho de trascendental importancia para la ciencia abierta (Higman, Bangert y Jones, 2019; Mons, 2018; Barba, 2018; Plotkin, 2014). Asimismo, surgen las figuras del bibliotecario de datos (data librarian) y del administrador de datos (data steward) como agentes propulsores de nuevos servicios para preservar los datos de investigación, garantizar la sostenibilidad de los sistemas de almacenamiento y la reusabilidad de los datos, y cumplir con las directrices de las agencias de financiación de la investigación.

Dado lo anterior, en este artículo se pretende ahondar en las siguientes preguntas:

1. ¿Qué servicios de GDI ofrecen las bibliotecas universitarias y de investigación en Europa y América Latina?

2. ¿Qué tareas se les atribuyen a los bibliotecarios y administradores de datos en el contexto académico?

Para ello, la estructura consiste en cinco secciones. Tras esta sección, donde se introduce el tema y se presentan las preguntas de investigación, la segunda sección contextualiza la GDI y su vínculo con las bibliotecas universitarias y de investigación. La tercera sección explica la metodología. La cuarta sección presenta los resultados sobre los servicios bibliotecarios para GDI. La quinta sección concluye el artículo y delinea posibles trabajos futuros.

\section{La gestión de los datos de investigación}

La GDI está presente en el ciclo de vida de los datos y cubre los procesos de planificación, recopilación, selección, transformación y archivo de datos de investigación, siempre con el objetivo de almacenarlos a largo plazo y de forma independiente del creador de los datos, es decir, en una infraestructura 
externa (Whyte y Tedds, 2011).

Las demandas relacionadas con GDI varían mucho de un área de investigación a otra y también entre los agentes involucrados en el proceso. Los investigadores a menudo tienen necesidades e ideas diferentes a las de un organismo de financiación o un socio de cooperación de la industria, dependiendo de la fase de investigación y del tipo de datos generados.

Idealmente, las prácticas de GDI deben comenzar con un plan de gestión de datos (PGD, del inglés Data Management PlanDMP) que registra, el tipo de datos generados y reutilizados, los formatos, estándares y métodos de captura, así como las implicaciones éticas y las estrategias de compartición y preservación (Peset Mancebo y Gonzalez, 2017). Un PGD ayuda a planificar un proceso de investigación y a definir responsabilidades en un proyecto de investigación en el que participan varios investigadores o instituciones. Esto también permite identificar el aumento de las necesidades de recursos en una fase temprana.

Los PGDs son requeridos por una gran parte de las agencias de financiamiento y cada vez hay más iniciativas para mejorar su legibilidad por máquinas, de forma que los investigadores solo deban completar un mínimo de preguntas y el resto de la información sea recuperada de otras infraestructuras (p.ej. repositorio, bancos de publicaciones o ORCID) (Miksa, Simms, Mietchen y Jones, 2019; Miksa, Rauber, Ganguly y Budroni, 2017).

Cabe señalar que el concepto de GDI coexiste con otros dos conceptos: los principios FAIR y los datos abiertos (Higman et al., 2019). Los principios FAIR abogan por un mayor acceso de los datos, metadatos y otros resultados de la investigación. Por su parte, los datos abiertos son datos disponibles sin restricciones o con restricciones mínimas. Si un conjunto de datos abierto está bien documentado y es legible por máquinas, tiene una licencia abierta (p.ej. CCO o CC-BY), utiliza formatos y estándares abiertos independientes del proveedor, estos datos abiertos son datos FAIR.

Varios estudios reconocen que las bibliotecas universitarias y de investigación se encuentran en una posición ideal para asistir a los investigadores durante todo el ciclo de vida de su investigación, ya que las necesidades de apoyo en GDI se pueden suplir mejor cuando la información sobre los servicios es gestionada de forma centralizada (Cox, Kennan, Lyon, Pinfield y Sbaffi, 2019; Tripathi, Shukla y Sonkar, 2017; Tenopir et al., 2017; Briney, 2015; Ray, 2014; Tenopir, Sandusky, Allard y Birch, 2014).

La siguiente figura ejemplifica cómo las instituciones pueden distribuir los servicios de GDI según ciclo de vida de los datos, con enfoque central en el investigador. Este modelo evidencia la continuidad de vida de los datos en otras investigaciones, independientemente de la finalización de su uso por el proyecto que los genera.

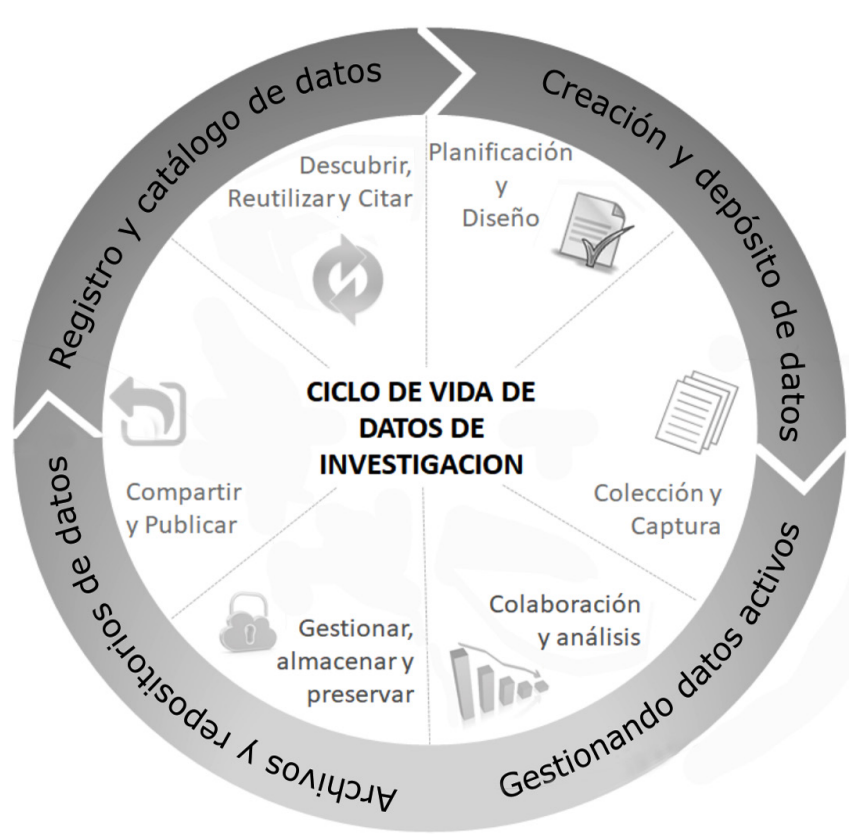

Figura 1. Ciclo de vida de los datos de investigación (traducción propia). Fuente: JISC, 2016.

Los modelos de ciclo de vida ayudan a definir e ilustrar procesos complejos, lo que facilita la identificación de las partes componentes o las distintas etapas de los datos de investigación y sus relaciones directas con los servicios de datos.

Vincular los servicios individuales e identificar cómo se encajan en un marco holístico mayor, ayuda a identificar posibles brechas y deficiencias en los servicios (Rice y Southall, 2016; Ray, 2014).

Es importante resaltar que las buenas prácticas en la gestión de datos varían en función de las colecciones de datos de investigación, la naturaleza del contexto, el contenido y el formato de los datos existentes y su relación con el ambiente tecnológico de la institución.

\section{Procedimientos metodológicos}

La metodología de este trabajo es cualitativa y se encaja dentro de una investigación exploratoria que pretende dar una visión general sobre el tratamiento de los datos dentro de las bibliotecas europeas y latinoamericanas, así como sobre las habilidades requeridas para las profesiones de bibliotecario de datos (data librarian) y administrador especialista de datos (data steward).

En primer lugar, se realiza un análisis bibliográfico en diferentes bases de datos y buscadores académicos con las palabras clave «gestión de datos de investigación» y «bibliotecas» 
y en inglés, español y portugués. De entre los documentos recuperados se han tenido principalmente en cuenta los artículos y los informes y/o guías redactados por asociaciones de bibliotecas, por ejemplo, la Liga de Bibliotecas Europeas de Investigación (LIBER). Junto con el análisis bibliográfico, se realiza un mapeo de los servicios de GDI en 34 bibliotecas universitarias y de investigación europeas y latinoamericanas?.

Dada las características de los estudios cualitativos, la selección de la muestra no responde a criterios probabilísticos, por tanto, su número se debe a la saturación de casos y la capacidad de recolección y análisis para el entendimiento del fenómeno (Hernández Sampieri, Fernández Collado y Baptista Lucio, 2010).

Esto lleva a considerar que los casos de selección de esta muestra se basen según el retorno dado por los algoritmos de los sistemas de clasificación del buscador general de Google. Los mencionados algoritmos responden a un conjunto de factores como las palabras de la consulta empleada ("gestión de datos de investigación"+ "país2"), la relevancia y usabilidad de las páginas, la ubicación3 desde la cual se realizo la búsqueda, entre otros. No se establecieron ajustes a la configuración del buscador que incidiera en los resultados obtenidos.

Como resultado de esta búsqueda se obtuvo el siguiente número de bibliotecas por sus respectivos países (ver tabla 1).

Además, se incorporan elementos comparativos para la fundamentación de la situación de la región de América Latina (estado de São Paulo de Brasil, Argentina, México, Perú, etc.). Excluyendo Chile, no se encontraron páginas informativas sobre servicios de GDI en países latinoamericanos, por lo que no pasaron a formar parte de la muestra seleccionada. No obstante, sí que se encontraron iniciativas a nivel nacional o

\begin{tabular}{|l|c|}
\hline \multicolumn{1}{|c|}{ País } & número de bibliotecas \\
\hline Alemania & 9 \\
\hline Reino Unido & 8 \\
\hline Irlanda & 4 \\
\hline España & 3 \\
\hline Suiza & 2 \\
\hline Bélgica & 1 \\
\hline Chile & 1 \\
\hline Dinamarca & 1 \\
\hline Finlandia & 1 \\
\hline Noruega & 1 \\
\hline Países Bajos & 1 \\
\hline Suecia & 1 \\
\hline Indefinido ${ }^{4}$ & 1 \\
\hline
\end{tabular}

Tabla 1. Número de bibliotecas por paises. Elaboración propia. estatal en vários países. Estas iniciativas fueron incorporadas como elementos comparativos e indicadores de futuras líneas de desarrollo. Asimismo, como elemento comparativo y complementario, se utilizó el registro de miembros de $\mathrm{ORCID}^{5}$ y de DataCite ${ }^{6}$ para analizar la implicación de las bibliotecas en el uso y registro de identificadores persistentes, especialmente para datos. Esta inclusión se realizó más tarde en el análisis, ya que se observó la alta implicación de las biblioteca en el registro de indentificadores persistentes.

Los principales elementos distintivos del mapeo de las bibliotecas que forman parte de la muestra se describen en la tabla 2. Además, este mapeo identifica además los servicios que las bibliotecas prestan en cada etapa del ciclo de investigación según la información que aparece en sus páginas web o en publicaciones al respecto.

En la tabla 3 se describen los valores de análisis aplicados al criterio de descripción servicios.

Al unir estas dos etapas se establece el marco teórico del trabajo del bibliotecario de datos y el administrador especialista de datos, así como la actuación de las bibliotecas en la gestión de los datos como bienes científicos para el futuro.

Como indicado por LIBER (Christensen-Dalsgaard et al., 2012), las bibliotecas desempeñan un papel relevante, a veces incluso de coordinación, en el desarrollo de servicios para GDI. Sin embargo, a pesar de su implicación en estas tareas, las bibliotecas actúan en colaboración con otras unidades universitarias, especialmente en aspectos informáticos o asesoría para proyectos financiados con fondos públicos (Tenopir et al., 2017).

Cabe añadir que al planificar servicios de GDI es importante proceder con un claro enfoque en la gobernanza de datos y estableciendo flujos de trabajo y responsabilidad, ya que suele haber diferentes unidades involucradas. Rice y Southall (2016) señalan la desinformación de los responsables por la GDI sobre la comunidad de investigadores; la falta de adecuación de la oferta a la satisfacción de las necesidades y expectativas de sus usuarios y la ausencia de compromiso sobre los servicios de GDI por parte de las unidades responsables como motivo para el rechazo o poca aceptación de los nuevos servicios.

El estudio expone los resultados del mapeo de los servicios de GDI en Europa y Latinoamérica a partir de las fuentes de información consultadas, que se limitan a la información pública accesible en las páginas web de las bibliotecas.

\section{Servicios bibliotecarios para la gestión de datos de investigación}

Dado el rol central de la gobernanza de datos en los procesos de GDI, se destaca el desarrollo de políticas institucionales de 


\begin{tabular}{|c|c|c|c|c|c|c|c|}
\hline & $\begin{array}{c}\text { Universidad o } \\
\text { Institución }\end{array}$ & $\begin{array}{c}\text { País de Proce- } \\
\text { dencia }\end{array}$ & $\begin{array}{c}\text { Página web } \\
\text { GDI }\end{array}$ & $\begin{array}{c}\text { Ubicación den- } \\
\text { tro de la página } \\
\text { web institucio- } \\
\text { nal }\end{array}$ & Servicios & $\begin{array}{c}\text { Política } \\
\text { GDI }\end{array}$ & $\begin{array}{c}\text { Enlace web } \\
\text { de la Política } \\
\text { de GDI }\end{array}$ \\
\hline \multirow{2}{*}{ Valores } & $\begin{array}{c}\text { nombre } \\
\text { próprio } \\
\text { normalizado }\end{array}$ & Nombre & URL & $\begin{array}{c}\text { Jerarquia de } \\
\text { clasificación } \\
\text { dentro de la } \\
\text { página web } \\
\text { institucional }\end{array}$ & $\begin{array}{c}\text { Listado de } \\
\text { servicios }\end{array}$ & Sí/No & URL \\
\hline
\end{tabular}

Tabla 2. Criterios de descripción de la GDI. Elaboración propia.

\begin{tabular}{|c|c|}
\hline Criterios de análisis & Descripción \\
\hline $\begin{array}{l}\text { Información sobre planes de ges- } \\
\text { tión de datos }\end{array}$ & $\begin{array}{l}\text { - Creación, alcance, equipo de trabajo dentro y fuera de la biblioteca, información sobre } \\
\text { sus objetivos y metas, contatos } \\
\text { - Funcionamento de trabajo con los datos, público objetivo } \\
\text { - Existencia del rol de Data Champions }\end{array}$ \\
\hline Tipos de servicios ofrecidos & 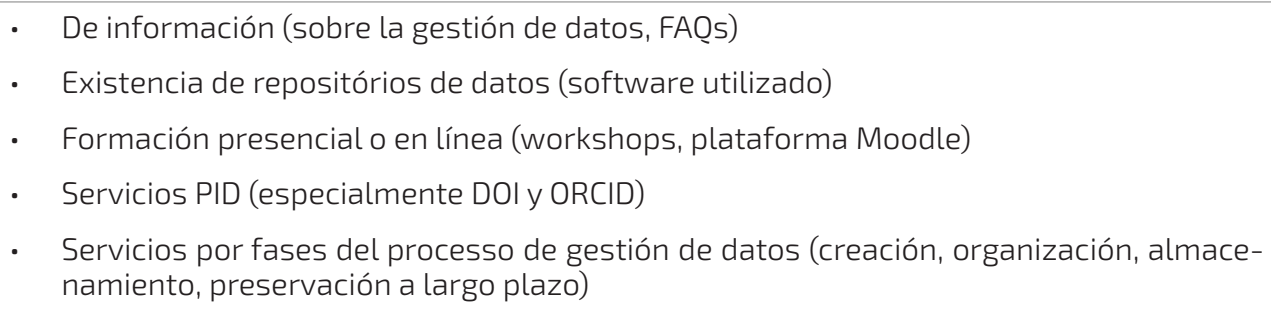 \\
\hline $\begin{array}{l}\text { Recursos de información y } \\
\text { herramientas tecnológicas dispo- } \\
\text { nibilizadas }\end{array}$ & $\begin{array}{l}\text { - Guías de usuarios (p.ej. sobre elaboración de una estrategia de GDI, sobre ORCID) } \\
\text { - Herramientas disponibilizadas para el trabajo individual y colaborativo } \\
\text { - Información sobre las directrices de trabajo }\end{array}$ \\
\hline Aspectos legales & $\begin{array}{l}\text { - Información sobre el soporte legal, normativa vigente, licencias } \\
\text { - Inclusión de la información sobre los derechos de autor en los metadatos dispo- } \\
\text { nibilizados por el repositorio de datos }\end{array}$ \\
\hline
\end{tabular}

Tabla 3. Criterios de análisis de los servicios. Elaboración propia.

gestión de datos. Veinticuatro (24) de las treinta y cuatro (34), un $70,5 \%$, instituciones analizadas poseen una política al respecto y el texto es de fácil acceso desde la página que detalla los servicios de GDI.

Algunas instituciones no desarrollan una política, sino que adoptan una política nacional. La mayoría de los países latinoamericanos se adhieren más a la centralización de las políticas y las infraestructuras. Algunos ejemplos son el desarrollo de una política de GDI por parte del Comisión Nacional de Investigación Científica y Tecnológica (CONICYT) en Chile7, la Resolución 753 - E/2016 del Ministerio de Ciencia Argentino $^{8}$ que reglamenta la ley de creación de repositorios digitales institucionales de acceso abierto, la creación de Consórcio Nacional em Educação, Ciência, Tecnologia e Inovação (CONECTI) en Brasil con un enfoque en la integración de ORCID, o las nuevas implementaciones en la gestión de datos en el Repositorio Nacional Digital de Ciencia, Tecnología e Innovación (ALICIA ${ }^{10}$ ) por parte de Consejo Nacional de Cien- cia, Tecnología e Innovación Tecnológica (CONCYTEC) en Perú.

\subsection{Asesoría y formación en gestión de datos de investigación}

Junto con la información inicial disponible en sus webs, las bibliotecas centran sus servicios de GDI en la asesoría en las diferentes partes del proyecto científico. Para abarcar mejor las necesidades institucionales en GDI, algunas bibliotecas establecen grupos de trabajo multidisciplinares que cuentan con la participación de representantes de diferentes facultades. Estos grupos de trabajo se han implementado en universidades como la Universidad de Estocolmo (Suecia) y la Universidad Técnica de Darmstadt (Alemania). Otro enfoque en ese sentido es el trabajo con data champions, investigadores con cierto prestigio que son ejemplares en la GDI. De esta forma, las bibliotecas de universidades como Cambridge (Reino Unido) o Delft (Países Bajos) consiguen tener «embajadores» de buenas prácticas en GDI que pueden dialogar 
directamente con otros investigadores de su área.

Además, la mayoría (19 de 34) de las bibliotecas analizadas ofrece formación para los investigadores en forma de talleres presenciales o virtuales durante el año. La Universidad de Southampton (Reino Unido), la Universidad de Delft y la Universidad Leibniz Hannover (Alemania) centran su oferta en jóvenes investigadores (doctorandos). A su vez, tanto la Universidad de Delft como la Universidad de Oslo han adoptado la metodología de talleres prácticos de «The Carpentries» ${ }^{11}$ para la enseñanza de la habilidades básicas en gestión de datos (Data Carpentry) y uso de software (Software Carpentry) para fines de investigación.

También es común realizar capacitaciones para otros bibliotecarios o personal que vaya a trabajar con GDI. Destacamos aquí el proyecto FDMentor ${ }^{12}$, financiado por el Ministerio de Educación e Investigación Alemán y llevado a cabo por la Universidad Humboldt de Berlín, la Universidad Libre de Berlín, la Universidad Técnica de Berlín y la Universidad de Potsdam, que entre sus objetivos principales tuvo la creación de competencias en GDI a través de un programa de formación de formadores (Train-the-Trainer) y del desarrollo de materiales de formación en lengua alemana.

\subsection{Herramientas para la generación de planes de gestión de datos}

Los PGD son documentos vivos que describen la gestión de los datos a lo largo de su ciclo de vida. Según la Comisión Europea, un plan de gestión de datos debe incluir la siguiente información: (1) el tratamiento de los datos de investigación durante y tras el proyecto; (2) el tipo de datos que será colectado, procesado y/o generado; (3) la metodología y estándares que se utilizarán; (4) si los datos se compartirán o disponibilizarán en acceso abierto; (5) cómo se realizará la curaduría y preservación de los datos.

Los PGDs son requeridos en proyectos europeos (por ejemplo, Horizon 2020 o ECR), así como en proyectos financiados por la FAPESP (Fundação de Amparo à Pesquisa do Estado de São Paulo) en Brasil o el CONICET (Consejo Nacional de Investigaciones Científicas y Técnicas) en Argentina.

Sin embargo, la creación de planes de datos se observa entre la comunidad científica como una actividad que consume mucho tiempo (Williams, Bagwell y Nahm Zozus, 2017). Por eso, han surgido diferentes herramientas online para la generación de planes de gestión de datos como DMPonline ${ }^{13}$ del Digital Curation Centre (DCC) o Datastewarship Wizard ${ }^{14}$, esta última con un enfoque a los PGD legibles por máquinas.

Las actividades de conceptualización, coordinación y gestión de este tipo de herramientas forman parte del trabajo de muchas bibliotecas en diferentes instituciones, así como la asesoría para investigadores en su uso. Destacamos las acti- vidades de la biblioteca de la Universidad de Gante (Bélgica) con DMPBelgium ${ }^{15}$; la biblioteca de la Universidad de Helsinki (Finlandia) con DMPTuuli'í; el conjunto de bibliotecas universitarias de la Comunidad de Madrid (España) con PGDOnline ${ }^{17}$ y las bibliotecas universitarias de Cataluña con Pla de Gestió de Dades de Recerca ${ }^{18}$, todas ellas basadas en DMPonline.

En Alemania, la herramienta de preferencia es RDMO ${ }^{19}$ (Research Data Management Organizer) adaptada al contexto de cada universidad. Por ejemplo, la Universidad Técnica de Darmstadt ofrece TUdmo ${ }^{20}$, gestionada por el equipo TUdata ubicado en la biblioteca.

Otras instituciones han desarrollado herramientas propias basadas en código libre, como la Universidad Técnica de Berlín y TUB-DMP21.

Por su parte, las universidades públicas del estado de São Paulo (Universidade Estadual Paulista -UNESP, Universidade de São Paulo - USP y Universidade Estadual de Campinas UNICAMP), siguiendo la petición de la agencia de financiación FAPESP, han creado perfiles institucionales en la herramienta DMPTool22 de la California Digital Library.

\subsection{Repositorios de datos}

Diversas universidades disponibilizan algún tipo de sistema de información para preservar y difundir los resultados de investigación de la institución y, cada vez más, esos resultados incluyen conjuntos de datos. Las bibliotecas universitarias suelen participar directamente en el desarrollo conceptual (tipo de recursos aceptados, metadatos, flujos de trabajo internos, etc.) y en el mantenimiento de los repositorios.

En el análisis se percibió que muchas instituciones no poseen aún un repositorio específico para datos. Por eso, las páginas web de las bibliotecas recomiendan repositorios generales como Zenodo o acudir al registro re3data23 para encontrar un repositorio temático adecuado. Asimismo, varias páginas de bibliotecas hacen hincapié en la búsqueda de repositorios confiables certificados. Una de las certificaciones mencionada es CoreTrustSeal24, obtenida por 63 repositorios a nivel mundial (dato de agosto de 2019) entre los que se encuentra el Digital Repository of Ireland (DRI). En España, el repositorio Digital.CSIC también posee una certificación de confiabilidad, Data Seal of Approval (DSA), actualmente reemplazada por CoreTrustSeal.

Es importante señalar que la mayoría de los repositorios de datos institucionales es de reciente creación, debido a la actual demanda de apertura de datos científicos. Por ejemplo, el repositorio de datos de la University College London (Reino Unido) se inauguró en junio de 2019 y DepositOnce, de la Uni- 
versidad Técnica del Berlín, en el último semestre de 2018.

Otra tendencia es la creación de interfaces federadas que agrupen varios repositorios. Por ejemplo, e-cienciaDatos25, promovido por el Consorcio Madroño que incluye los repositorios de datos de las universidades de la comunidad de Madrid; - 4TU26, para las universidades técnicas neerlandesas. Asimismo, existen sistemas como RADAR27, gestionado por la TIB, que utilizan varias localizaciones para las copias de seguridad y ofrecen servicios para instituciones e investigadores.

En Brasil, se destaca el enfoque en la preservación de datos adoptado por la Rede Cariniana del Instituto Brasileño de Información en Ciencia y Tecnología (IBICIT) y su biblioteca. Este instituto ofrece un repositorio de datos ${ }^{28}$ basado en el software Dataverse. En la actualidad cuenta con más de 200 registros de datos identificados de forma persistente. A nivel de las bibliotecas universitarias, muchas están considerando la ampliación de sus repositorios para aceptar datos, como ocurre con el repositorio institucional de la Universidade Estadual Paulista (UNESP) basado en DSpace. A veces, las demandas de los investigadores son más rápidas que los avances institucionales. Por eso, algunos grupos de investigación deciden desarrollar soluciones a menor escala se producen. Un ejemplo es el proyecto piloto de sistema CRIS (Current Research Information System) para uno de los grupos del departamento de Ciencia de la Información de la UNESP (Vidotti et al., 2018).

\subsection{Servicios de registro de identificadores persistentes}

Los identificadores persistentes (Persistent Identifier - PIDs) son uno de los puntos principales para garantizar la encontrabilidad de los datos, como se refleja en el principio F1 de los principios FAIR ${ }^{29}$ : «A los (meta)datos se les asigna un identificador persistente globalmente único». También el proyecto europeo FREYA ${ }^{30}$ tiene entre sus objetivos que los identificadores persistentes formen parte de la investigación abierta.

Los PIDs pueden asignarse a agentes, recursos, declaraciones de derechos, eventos o entidades derivadas (Dappert, Farquhar, Kotarski y Hewlett, 2017). Asimismo, permiten la conexión e interoperabilidad entre todas las entidades, facilitando el descubrimiento de objetos digitales y su correspondiente atribución a sus creadores (Klein y Van de Sompel, 2017).

La gran mayoría de los repositorios institucionales actuales utiliza algún tipo de sistema de PIDs para sus registros. Generalmente, este sistema es el sistema Handle. Otros optan por los identificadores de objeto digital (Digital Object Identifier - DOI) o combinan ambos sistemas dependiendo del recurso que traten. De las bibliotecas analizadas, solo la de RWTH Aachen utiliza un sistema de PID diferente, ePIC. No obstante, tanto DOI como ePIC se basan en la arquitectura del sistema
Handle.

Para poder asignar DOls específicamente a conjuntos de datos, se creó en 2009 la agencia de registro DataCite. Desde entonces, varias bibliotecas universitarias y de investigación se han unido a DataCite para registrar DOls y convertirse centro de asignación de DOls, algunos de ellos a nivel nacional. Como ejemplo tenemos la Biblioteca Nacional Alemana de Ciencia y Tecnología, la biblioteca universitaria de la ETH Zürich (Suiza), la biblioteca de la Universidad de Göttingen (Alemania) o la biblioteca de la Universidad Técnica de Viena (Austria).

Otro ejemplo es el servicio nacional de registro de DOI de Portugal que, aunque está coordinado por la Fundación de Ciencia y Tecnología, cuenta con la participación de los servicios de documentación de la Universidad de Minho y se orienta a la red de Repositorios Científicos de Acceso Abierto de Portugal (RCAAP) ${ }^{31}$ (Marques, Carvalho, Lopes y Mesquita, 2018).

Como PID para investigadores, el ORCID iD se ha establecido como un estándar. Para poder modificar y actualizar la información contenida en el registro asociado a cada ORCID iD, las instituciones pueden optar por una membresía institucional o mediante un consorcio. En Europa, existen actualmente doce consorcios, cinco de los cuales -Austria, Alemania, Dinamarca, Grecia y Portugal-están coordinados por bibliotecas universitarias o de investigación. Al margen de la coordinación de los consorcios, las bibliotecas llevan a cabo una gran parte de la integración de ORCID en los sistemas institucionales (repositorios, sistemas de gestión de publicaciones, herramientas de PGD, sistemas CRIS, etc.).

Como ejemplos tenemos la integración de ORCID en todos los sistemas CRIS, basados en PURE de Elsevier, en Dinamarca, y el intercambio de datos entre el registro de control de autoridades (Gemeinsame Normdatei - GND) de la Biblioteca Nacional Alemana y ORCID, así como la implementación de ORCID en el motor de búsqueda BASE gestionado por la biblioteca de la Universidad de Bielefeld (Alemania).

En América Latina, el único consorcio está ubicado en Brasil y está compuesto, entre otros, por la biblioteca científica SciELO (Scientific Electronic Library Online), cuyo objetivo será la integración del sistema ORCID en la biblioteca en línea para contribuir a una mayor visibilidad de las investigaciones.

En México, Redalyc ${ }^{32}$ también intercambia sus datos con ORCID. A su vez, Redalyc es uno de los principales actores en AmeliCA ${ }^{33}$ y trabaja activamente para la construcción de infraestructuras científicas abiertas para la producción cien- 
tífica en el Sur Global.

Por otro lado, Perú permite a sus investigadores vincular sus publicaciones en el repositorio ALICIA con ORCID.

\subsection{Integración de los profesionales de datos}

La integración de las figuras del bibliotecario y del administrador de datos es un avance en las bibliotecas universitarias para la gestión de datos. Los bibliotecarios de datos son profesionales que se dedican completamente al trabajo con datos dentro de la biblioteca y a la orientación de investigadores en tareas de GDI (Springer, 2019). Es posible encontrar otros profesionales de las bibliotecas, como los especialistas en metadatos y comunicación científica que ejerzan a veces tareas de GDI.

Los administradores de datos se establecen de forma similar a los bibliotecarios especializados (liaison librarians) pero concentrándose en la GDI para esa disciplina. La biblioteca de la
Universidad Técnica de Delft (Países Bajos) ha sido pionera en la integración de esta figura. Un ejemplo similar se encuentra en la Universidad de Essex (Reino Unido) que integra Research Development Managers en los flujos de trabajo con datos en cada facultad. Por su parte, la Universidad Humboldt de Berlín (Alemania) y la University College Dublin (Irlanda) tienen a bibliotecarios de datos como parte de su plantilla.

Las tareas y competencias de ambos profesionales se presentan en la tabla 4.

En muchos casos, la formación necesaria para llevar a cabo estas tareas no forma parte de los programas de estudios de Biblioteconomía, Archivística, Documentación y Ciencia de la Información.

Como indican Schmidt, Calarco, Kuchma y Shearer (2016), cada institución desarrolla estrategias diferentes para formar a su personal que pueden variar desde cursos formales y certificados en formato presencial o virtual, talleres, conferencias o participación en grupos de trabajo ${ }^{34}$.

\begin{tabular}{|c|c|c|}
\hline & Bibliotecario de datos & Administrador de datos \\
\hline $\begin{array}{c}\text { Competencias y } \\
\text { tareas }\end{array}$ & $\begin{array}{l}\text { - Diseño y desarrollo conceptual de sistemas } \\
\text { (conocimiento de tecnología de metadatos, } \\
\text { protocolos infraestructuras digitales } \\
\text { de investigación, gobernanza, curaduría } \\
\text { digital, etc.) (Rice y Southall, 2016). } \\
\text { - } \quad \text { Dominio de los ecosistemas de investigación } \\
\text { (Kennan, 2016; Xia y Wang, 2014). } \\
\text { - Conocimiento y aplicación de los flujos } \\
\text { de trabajo en infraestructuras digitales } \\
\text { (Rice y Southall, 2016; Xia y Wang, 2014). } \\
\text { - Conocimiento de tecnologías de la información } \\
\text { y familiaridad con lenguajes de programación } \\
\text { para datos (p.ej. R y Python) (Bradley- } \\
\text { Ridout, 2018; Federer, 2018; Kennan, 2016). } \\
\text { - Conocimiento de métricas para } \\
\text { infraestructuras de investigación } \\
\text { (Federer, 2018). } \\
\text { Conocimiento de GDI (Federer, 2018; Kennan, } \\
\text { 2016; Rice y Southall, 2016; Snipes, 2018; } \\
\text { Tenopir et al., 2014; Xia y Wang, 2014). } \\
\text { Conocimiento de catálogos de sistemas y } \\
\text { datos (calidad de metadatos, semántica } \\
\text { y estándares) (Kennan, 2016). } \\
\text { Habilidades comunicativas y didácticas } \\
\text { (Kennan, 2016; Raju, 2014). }\end{array}$ & $\begin{array}{l}\text { - Asesoría específica y formación para } \\
\text { investigadores sobre GDI (Verheul et al., 2019). } \\
\text { - Conocimiento de las políticas de gestión de } \\
\text { datos y desarrollo de una específica para la } \\
\text { facultad de su disciplina (Verheul et al., 2019). } \\
\text { - } \quad \text { Asesoría sobre la escritura de un plan de GDI } \\
\text { (Jansen, van den Berg, van Overveld y Boiten, } \\
\text { 2019; Verheul et al., 2019; Mons, 2018). } \\
\text { - Conocimiento de estándares de metadatos } \\
\text { y ontologías existentes en la disciplina } \\
\text { (Verheul et al., 2019; Mons, 2018). } \\
\text { Conocimiento de los aspectos legales } \\
\text { y éticos de la gestión de datos (Dijkers, } \\
\text { 2019; Jansen et al., 2019; Mons, 2018). } \\
\text { Quehacer directo con nuevos flujos de trabajo } \\
\text { y nuevos desarrollos de infraestructura para } \\
\text { el área (Dijkers, 2019; Verheul et al., 2019). }\end{array}$ \\
\hline Formación & $\begin{array}{l}\text { Biblioteconomía con especialización } \\
\text { en gestión de datos. } \\
\text { - Formación próxima a la gestión de datos } \\
\text { y conocimientos del mundo de las biblio- } \\
\text { tecas universitarias y de investigación. }\end{array}$ & $\begin{array}{l}\text { - Máster o doctorado en la disciplina } \\
\text { y profunda comprensión de la GDI } \\
\text { y las políticas al respecto. }\end{array}$ \\
\hline Unidad de trabajo & $\begin{array}{l}\text { - Biblioteca universitaria o de investigación } \\
\text { o centro de información. }\end{array}$ & - Facultad de su disciplina. \\
\hline
\end{tabular}

Tabla 4. Contraste de las competencias, formación y unidades de trabajo del bibliotecario y administrador de datos. Fuente: Elaboración propia a partir de diversos autores. 


\section{Consideraciones finales}

Como apuntan McKenzie y Martin (2016), las bibliotecas universitarias y de investigación se enfrentan a un nuevo paradigma de datos que ha cambiado la práctica científica y su

sistematización. Esta transformación implica un cambio de roles, nuevos roles y la necesidad de desarrollar nuevas capacidades por el bibliotecario, hacia la integración de las figuras del bibliotecario y del administrador de datos. De esta forma, las bibliotecas pueden pasar de un modelo basado en servicios a uno basado en coparticipación y colaboración con los investigadores, como ocurre en las universidades que integran grupos de trabajo multidisciplinares o trabajan con data champions. Concretamente las bibliotecas desempeñarían un papel más proactivo en el proceso de creación de conocimiento y se ubicarían en cada fase del proceso de GDI, creando estrategias para la preservación de los datos a largo plazo.

Estos cambios se han podido observar en este mapeo inicial de servicios bibliotecarios, cuyo objetivo es centrarse en las demandas emergentes para la preservación de los resultados de investigación.

Aunque el estudio fue realizado solo sobre 34 páginas web de bibliotecas y publicaciones al respecto, fue posible extrapolar algunas conclusiones. La mayoría de las bibliotecas parte de servicios de información, formación y asesoría, como se observó siguiendo los criterios expuestos en la tabla 2. No obstante, también participan activamente en el desarrollo de nuevos servicios y herramientas.

Destacamos también la necesidad e importancia de contar con estrategias, normas y acuerdos alineados que generen oportunidades de cambio dentro del ecosistema de investigación, las cuales garanticen la solución de sus principales problemas. La existencia de una infraestructura y servicios de apoyo a la investigación no son suficientes para garantizar un nivel efectivo en este acometido. Se plantea entonces la necesidad de proceder en dicha planificación con un enfoque en la gobernanza de datos. De hecho, destacamos el alto desarrollo de políticas institucionales sobre gestión de datos.

Otro aspecto destacable es que las estrategias de GDI en América Latina aún son incipientes y se basan más en propuestas nacionales que institucionales. No obstante, se perciben grandes avances apoyados por la Biblioteca CEPAL, así como por el proyecto LEARN (Leaders Activating Research Networks).

Si bien el presente estudio limita el mapeo de los servicios de GDI en Europa y Latinoamérica basado exclusivamente en la información disponible en las páginas web de las biblioteca, se platea el estudio directo siguiendo la metodología de encuesta para análisis futuros. Así se establecería un contacto directo con gestores de bibliotecas y se podría analizar mejor la colaboración interinstitucional y los planes futuros. Un ejemplo de este tipo de encuesta es el desarrollado por Cox, Kennan, Lyon, Pinfield y Sbaffi (2019). Además, dada la cantidad de estudios sobre las estrategias y prácticas GDI en Europa, América del Norte y Oceanía, se considera más relevante destacar iniciativas en América Latina y África (el denominado «Sur Global») para caracterizar mejor el desarrollo del GDI en estas zonas geográficas en las que han surgido recientemente iniciativas muy valiosas como AmeliCA o la African Open Science Platform35.

\section{Notas alfinal}

1 Los datos extraídos del análisis se encuentran en un archivo .ods complementario en el sistema de gestión de la revista.

2 En esta posición fueron alternándose diferentes países europeos para abarcar uma muestra representativa de posibles países.

3 Desde una estación de trabajo dentro del domínio AT que corresponde a Austria.

4 Corresponde con el análisis de la CEPAL, que abarca América Latina.

5 Registro de miembros de ORCID: https://orcid.org/members

6 Registro de miembros de DataCite: https://datacite.org/members. html

7 Política datos científicos abiertos (Chile): http://datoscientificos. $\mathrm{cl} /$ politica

8 Texto de la resolución: https://www.argentina.gob.ar/normativa/ nacional/resoluci\%C3\%B3n-753-2016-267833

9 CONECTI: https://www.conectibrasil.org/

10 ALICIA: https://alicia.concytec.gob.pe/

1 The Carpentries: https://carpentries.org/

12 El nombre FDMentor surge de la combinación de la abreviatura GDI en alemán FDM (Forschungsdatenmanegement) y la palabra «Mentor». Los resultados de este proyecto se encuentran en su comunidad de Zenodo: https://zenodo.org/communities/fdmentor

13 DMPOnline: https://dmponline.dcc.ac.uk/

14 DataStewardship Wizard: https://ds-wizard.org/

15 DMP Belgium: https://dmponline.be/

16 DMPTuuli: https://www.dmptuuli.fi/

17 PGD Consorcio Madroño: http://pgd.consorciomadrono.es/

18 Pla de Gestió de Dades de Recerca: https://dmp.csuc.cat/

19 RDMO: https://rdmorganiser.github.io/

20 TUdmo: https://tudmo.ulb.tu-darmstadt.de/

21 TUB-DMP: https://dmp.tu-berlin.de/ y su código https://github. com/tuub/TUB-DMP

22 DMPTool: https://dmptool.org/

23 re3data: https://www.re3data.org/

24 CoreTrustSeal: https://www.coretrustseal.org/

25 e-ciencia datos: https://edatos.consorciomadrono.es/

26 4TU https://researchdata.4tu.nl/en/

27 RADAR: https://www.radar-service.eu/en

28 IBICT Dataverse: https://repositoriopesquisas.ibict.br/

29 Principio FAIR F1: https://www.go-fair.org/fair-principles/f1-meta-data-assigned-globally-unique-persistent-identifiers/ 
30 Proyecto FREYA: https://www.project-freya.eu/en/about/mission

31 RCAAP: https://www.rcaap.pt/

32 Redalyc: https://www.redalyc.org/home.oa

33 AmeliCA: http://amelica.org/

$34 \mathrm{El}$ anexo 1 recoge algunas posibilidades de especializaciones y complementos de formación existentes. Puede servir de guía inicial de posibilidades para aquellas bibliotecas que piensen en ampliar las competencias de sus empleados $y$, consecuentemente, su portfolio de servicios.

35 African Open Science Platform: http://africanopenscience.org.za/

\section{Referencias}

Alonso-Arévalo, J. (2019). ¿Qué es y qué importancia tiene la gestión de datos de investigación (GDI)? DesiderataLAB, (10), 48-52. http:// hdl.handle.net/10366/139056

Amorim, R. C., Castro, J. A., Rocha da Silva, J., y Ribeiro, C. (2017). A comparison of research data management platforms: Architecture, flexible metadata and interoperability. Universal Access in the Information Society, 16(4), 851-862. https://doi.org/10.1007/s10209-0160475-y

Barba, L. A. (2018). Terminologies for Reproducible Research. arXiv:1802.03311 [cs]. Recuperado de http://arxiv.org/abs/1802.03311

Bradley-Ridout, G. (2018). Preferred but not Required: Examining Research Data Management Roles in Health Science Librarian Positions. Journal of the Canadian Health Libraries Association / Journal de l'Association des bibliothèques de la santé du Canada, 39(3), 138-145. https://doi.org/10.29173/jchla29368

Briney, K. (2015). Data management for researchers: Organize, maintain and share your data for research success. Exeter, UK: Pelagic Publishing.

Christensen-Dalsgaard, B., Grim, R., Van der Berg, M., Horstmann, W., Jansen, D., Pollard, T., y Roos, A. (2012). Ten recommendations for libraries to get started with research data management. Recuperado de https://libereurope.eu/wp-content/uploads/The\%20 research\%20data\%20group\%202012\%20v7\%20final.pdf

Corrall, S. (2014). Designing Libraries for Research Collaboration in the Network World: An Exploratory Study. LIBER Quarterly, 24(1), 17. https://doi.org/10.18352/lq.9525

Corrall, S., Kennan, M. A., y Afzal, W. (2013). Bibliometrics and Research Data Management Services: Emerging Trends in Library Support for Research. Library Trends, 61(3), 636-674. https://doi.org/10.1353/ lib.2013.0005

Cox, A., Kennan, M. A., Lyon, L., Pinfield, S., y Sbaffi, L. (2019). International survey of research data management in libraries [Data set] https://doi.org/10.15131/shef.data.9204509.v1

Dappert, A., Farquhar, A., Kotarski, R., y Hewlett, K. (2017). Connecting the Persistent Identifier Ecosystem: Building the Technical and Human Infrastructure for Open Research. Data Science Journal, 16. https:// doi.org/10.5334/dsj-2017-028

Dijkers, M. P. (2019). A beginner's guide to data stewardship and data sharing. Spinal Cord, 57(3), 169-182. https://doi.org/10.1038/s41393018-0232-6

Federer, L. (2018). Defining data librarianship: A survey of competencies, skills, and training. Journal of the Medical Library Association,

106(3). https://doi.org/10.5195/JMLA.2018.306
Hernández Sampieri, R., Fernández Collado, C., y Baptista Lucio, P. (2010). Metodología de la investigación. (6a edición) México: McGraw-Hill / Interamericana editores, S.A.

Hey, T., Tansley, S., y Tolle, K. (2009). The fourth paradigm: Data-intensive scientific discovery. Redmond, Washington: Microsoft Research.

Higman, R., Bangert, D., y Jones, S. (2019). Three camps, one destination: The intersections of research data management, FAIR and Open. Insights the UKSG Journal, 32(18). https://doi.org/10.1629/uksg.468

Higman, R., y Pinfield, S. (2015). Research data management and openness: The role of data sharing in developing institutional policies and practices. Program, 49(4), 364-381. https://doi.org/10.1108/ PROG-01-2015-0005

Jansen, P., van den Berg, L., van Overveld, P., y Boiten, J.-W. (2019). Research Data Stewardship for Healthcare Professionals. En P. Kubben, M. Dumontier, y A. Dekker (Eds.), Fundamentals of Clinical Data Science, 37-53. https://doi.org/10.1007/978-3-319-99713-1_4

Kennan, M. A. (2016). Data Management: Knowledge and skills required in research, scientific and technical organisations. Proceedings IFLA Conference 2016. Presentado en Colombus. Recuperado de http://library.ifla.org/1466/1/221-kennan-en.pdf

Klein, M., y Van de Sompel, H. (2017). Discovering Scholarly Orphans Using ORCID. arXiv:1703.09343 [cs]. Recuperado de http://arxiv.org/ abs $/ 1703.09343$

Kowalczyk, S., y Shankar, K. (2011). Data sharing in the sciences. Annual Review of Information Science and Technology, 45(1), 247-294. https://doi.org/10.1002/aris.2011.1440450113

Maredata (2018). Recomendaciones para la gestión de datos de investigación dirigidas a investigadores. Recuperado de https:// digital.csic.es/handle/10261/173801

Marques, C., Carvalho, J., Lopes, P., y Mesquita, N. (2018). Serviço Nacional de Registo de Identificadores DOI. Atas da $9^{a}$ Conferência Luso Brasileira sobre Acesso Aberto. Recuperado de https://www. bad.pt/publicacoes/index.php/cadernos/article/view/1914

McKenzie, A., y Martin, L. (Eds.). (2016). Developing digital scholarship: Emerging practices in academic libraries. London: Facet Publishing.

Miksa, T., Rauber, A., Ganguly, R., y Budroni, P. (2017). Information Integration for Machine Actionable Data Management Plans. International Journal of Digital Curation, 12(1), 22-35. https://doi.org/10.2218/ijdc. v12i1.529

Miksa, T., Simms, S., Mietchen, D., y Jones, S. (2019). Ten principles for machine-actionable data management plans. PLOS Computational Biology, 15(3), e1006750. https://doi.org/10.1371/journal.pcbi.1006750

Mons, B. (2018). Data Stewardship for Open Science: Implementing

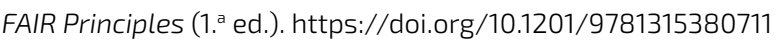

Perrier, L., y Barnes, L. (2018). Developing Research Data Management Services and Support for Researchers: A Mixed Methods Study. Partnership: The Canadian Journal of Library and Information Practice and Research, 13(1). https://doi.org/10.21083/partnership.v13i1.4115

Peset Mancebo, M. F., y Gonzalez, L.-M. (2017). Ciencia abierta y gestión de datos de investigación (RDM). Gijón: Trea.

Plotkin, D. (2014). Data stewardship: An actionable guide to effective data management and data governance. Amsterdam; Boston: Elsevier/Morgan Kaufman.

Raju, J. (2014). Knowledge and skills for the digital era academic library. The Journal of Academic Librarianship, 40(2), 163-170. https:// 
doi.org/10.1016/j.acalib.2014.02.007

Ray, J. M. (Ed.). (2014). Research data management: Practical strategies for information professionals. West Lafayette, Indiana: Purdue University Press.

Rice, R., y Southall, J. (2016). The data Librarian's handbook. London: Facet Publishing.

Schmidt, B., Calarco, P., Kuchma, I., y Shearer, S. (2016). Time to Adopt: Librarians' New Skills and Competency Profiles. Stand Alone, 1-8. https://doi.org/10.3233/978-1-61499-649-1-1

Snipes, G. (2018). Everyone's a data librarian now. Journal of New Librarianship, 3(1), 28-31. https://doi.org/10.21173/newlibs/4/6

Springer, R. (29 de julio de 2019). Counting Data Librarians [Ithaka S+R Blog website]. Recuperado de: https://sr.ithaka.org/blog/counting-data-librarians/

Tenopir, C., Sandusky, R. J., Allard, S., y Birch, B. (2014). Research data management services in academic research libraries and perceptions of Librarians. Library \& Information Science Research, 36(2), 84-90. https://doi.org/10.1016/j.lisr.2013.11.003

Tenopir, C., Talja, S., Horstmann, W., Late, E., Hughes, D., Pollock, D., Schmidt, B., Baird, L., Sandusky, R., y Allard, S. (2017). Research Data Services in European Academic Research Libraries. Liber Quarterly, 27(1), 23-44. https://doi.org/10.18352/lq.10180

Tripathi, M., Shukla, A., y Sonkar, S. K. (2017). Research Data Management Practices in University libraries: A study. DESIDOC Journal of Library \& Information Technology, 37(6), 417. https://doi. org/10.14429/djlit.37.6.11336

Verheul, I., Imming, M., Ringerma, J., Mordant, A., Ploeg, J.-L. van der, y Pronk, M. (2019). Data Stewardship on the map: A study of tasks and roles in Dutch research institutes. https://doi.org/10.5281/ zenodo.2669149

Vidotti, S. A. B. G., Coneglian, C. S., Roa-Martínez, S. M., Arakaki, F. A., Brandt, M. B., y Ferreira, A. M. J. F. da C. (2018). Repositório de dados de pesquisa para grupo de pesquisa: Um projeto piloto. Informação \& Tecnologia, 4(2), 221-242. https://doi.org/10.22478/ufpb. 2358-3908.2017v4n2.40198

Whyte, A., y Tedds, J. (2011). Making the Case for Research Data Management. DCC Briefing Papers. Edinburgh: Digital Curation Centre. Recuperado de http://www.dcc.ac.uk/resources/briefing-papers

Wilkinson, M. D., Dumontier, M., Aalbersberg, Ij. J., Appleton, G., Axton, M., Baak, A., y Mons, B. (2016). The FAIR Guiding Principles for scientific data management and stewardship. Scientific Data, 3, 160018. https://doi.org/10.1038/sdata.2016.18

Williams, M., Bagwell, J., y Nahm Zozus, M. (2017). Data management plans: The missing perspective. Journal of Biomedical Informatics, 71, 130-142. https://doi.org/10.1016/j.jbi.2017.05.004

Xia, J., y Wang, M. (2014). Competencies and Responsibilities of Social Science Data Librarians: An Analysis of Job Descriptions. College 8 Research Libraries, 75(3), 362-388. https://doi.org/10.5860/crl13-435

\section{CV}

Paloma Marín-Arraiza. Licenciada en Física, máster en Información y Comunicación Científica por la Universidad de Granada (España) y doctoranda en Ciencia de la Información en la Universidad Estatal Paulista (Brasil) con enfoque en publicaciones ampliadas (enhanced publications) y gestión de datos de investigación. Fue miembro del grupo de competencias de materiales no textuales en la Biblioteca Nacional Alemana de Ciencia y Tecnología (TIB Hannover), donde trabajó con metadatos para materiales audiovisuales y datos abiertos enlazados. En la actualidad es gestora de información con enfoque en identificadores persistentes en la biblioteca de la Universidad Técnica de Viena (TU Wien Bibliothek), donde se encarga del consorcio austriaco de ORCID y del servicio de registro de DOls. Filiación: Universidade Estadual Paulista (UNESP) y TU Wien Bibliothek. https://www.linkedin.com/in/ paloma-marin-arraiza/

Mirelys Puerta-Díaz. Llicenciada en Bibliotecología y Ciencias de la Información, máster en Bibliotecología y Ciencia de la Información por la Universidad de La Habana (Cuba) y doctoranda en Ciencia de la Información por la Universidad Estatal Paulista (Brasil) con enfoque en la publicación de datos abiertos enlazados, así como en las métricas y dinámicas de representación de la producción científica de la opinión pública. Profesora Asistente en la Facultad de Comunicación de la Universidad de La Habana. Filiación: Universidade Estadual Paulista (UNESP). https://www.linkedin.com/in/ mirelyspd/

Silvana Gregorio-Vidotti. Licenciada en Matemática por la Universidade Estadual Paulista - UNESP (Brasil), máster en Ciencias - Ciencias de la Computación y Matemática Computacional por la Universidade de São Paulo - USP (Brasil) y doctora en Educación por la UNESP. Lidera el grupo de investigación «Novas Tecnologias em Informação» y profesora en la Facultad de Ciências y Filosofia, dentro del departamento de Ciencia de la Información de la UNESP. Sus líneas e intereses de investigación son la arquitectura de la información y la experiencia de usuario en ambientes de información digitales. Filiación: Universidade Estadual Paulista (UNESP). http://lattes.cnpq.br/7390573927636069 


\section{Anexo 1: Formación en biblioteconomía de datos y gestión de datos de investigación}

\begin{tabular}{|c|c|}
\hline \multicolumn{2}{|r|}{ Oferta de formación en biblioteconomía de datos y GDI } \\
\hline \multicolumn{2}{|r|}{ Data librarian } \\
\hline Tipo & Especialización \\
\hline Institución & Escuela Técnica Superior de Colonia (TH Köln) \\
\hline Duración & 7 ECTS repartidos en 6 módulos y dos semestres \\
\hline Contenido & 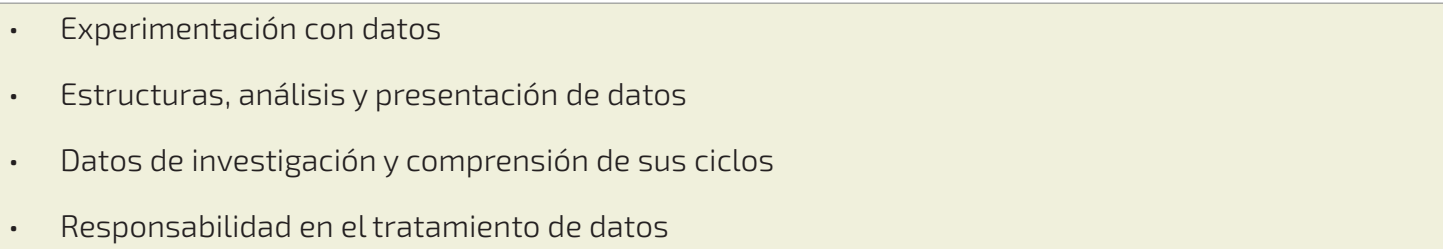 \\
\hline Enlace & https://www.th-koeln.de/weiterbildung/zertifikatskurs-data-librarian_63393.php \\
\hline \multicolumn{2}{|r|}{ Data librarian } \\
\hline Tipo & Especialización \\
\hline Institución & Universidad de Viena, Universidad de Graz y Universidad de Innsbruck \\
\hline Duración & 10 ECTS repartidos en tres módulos y dos semestres \\
\hline Contenido & 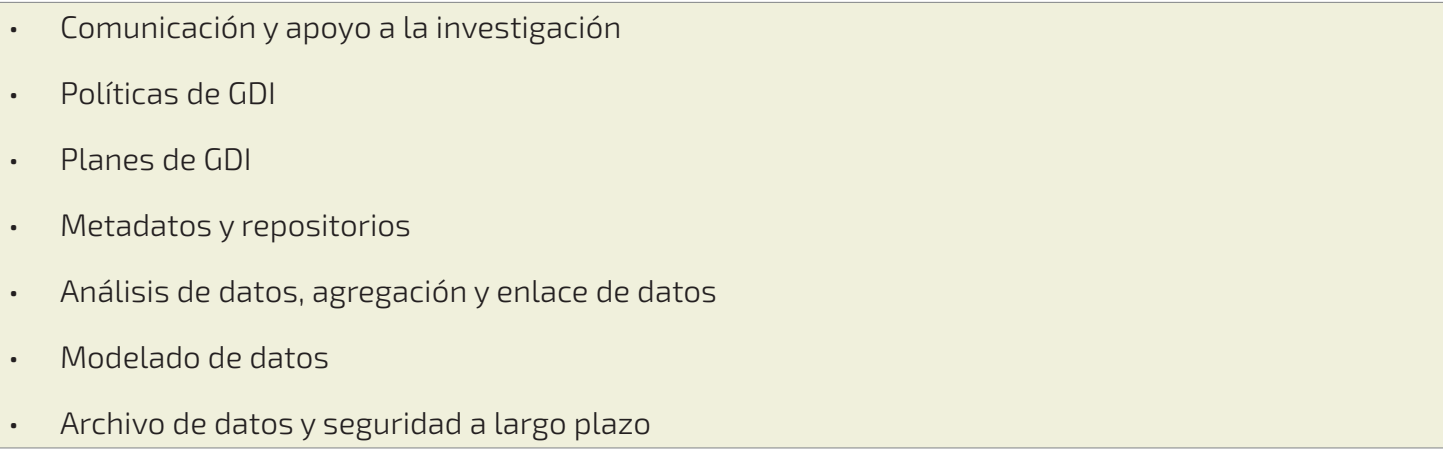 \\
\hline Enlace & https://www.postgraduatecenter.at/en/programs/communication-media/data-librarian/ \\
\hline \multicolumn{2}{|r|}{ Máster en Ciencia de datos } \\
\hline Tipo & Máster y complementos de formación \\
\hline Institución & Universidad Abierta de Cataluña (UOC) \\
\hline Duración & $\begin{array}{l}54 \text { ECTS de complementos de formación para el área de Información y Documentación + } 60 \text { ECTS pro- } \\
\text { pios del máster universitario }\end{array}$ \\
\hline Contenido & $\begin{array}{l}\text { Máster universitario: } \\
\text { - Recolección, manipulación y almacenamiento de datos (3 asignaturas) } \\
\text { - } \text { - Visuálisis de datos (7 asignaturas) } \\
\text { Complementón de datos (2 asignaturas) } \\
\text { - Estadística } \\
\text { - Álgebra } \\
\text { - Grafos y complejidad }\end{array}$ \\
\hline
\end{tabular}




\begin{tabular}{|c|c|}
\hline & 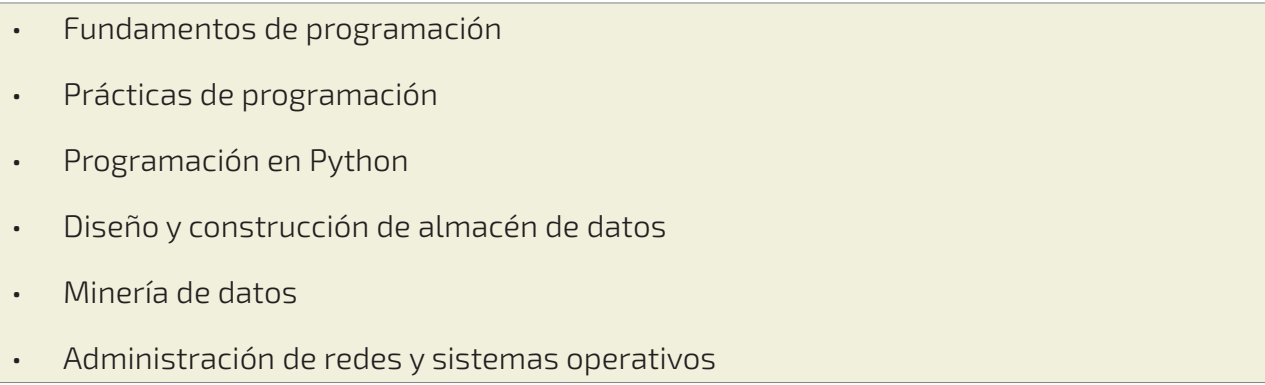 \\
\hline Enlace & https://estudios.uoc.edu/es/masters-universitarios/data-science/presentacion \\
\hline \multicolumn{2}{|r|}{ Workshop Library Carpentry } \\
\hline Tipo & Taller práctico \\
\hline Institución & Library Carpentry - The Carpentries \\
\hline Duración & 2 días ( $\approx 14 / 16$ horas) \\
\hline Contenido & $\begin{array}{l}\text { - Introducción a los datos } \\
\text { · UNIX Shell } \\
\text { - Open Refine } \\
\text { - Introducción a Git } \\
\text { - Tiny Data } \\
\text { - SQL } \\
\text { - Webscraping } \\
\text { - Introducción a Python } \\
\text { - Introducción a los datos para archivistas }\end{array}$ \\
\hline Enlace & https://librarycarpentry.org/ \\
\hline \multicolumn{2}{|r|}{ Delivering Research Data Management Services } \\
\hline Tipo & MOOC - Future Learn \\
\hline Institución & Universidad de Edimburgo, Digital Curation Centre y Research Data Netherlands \\
\hline Duración & 5 semanas ( $\approx 15$ horas) \\
\hline Contenido & 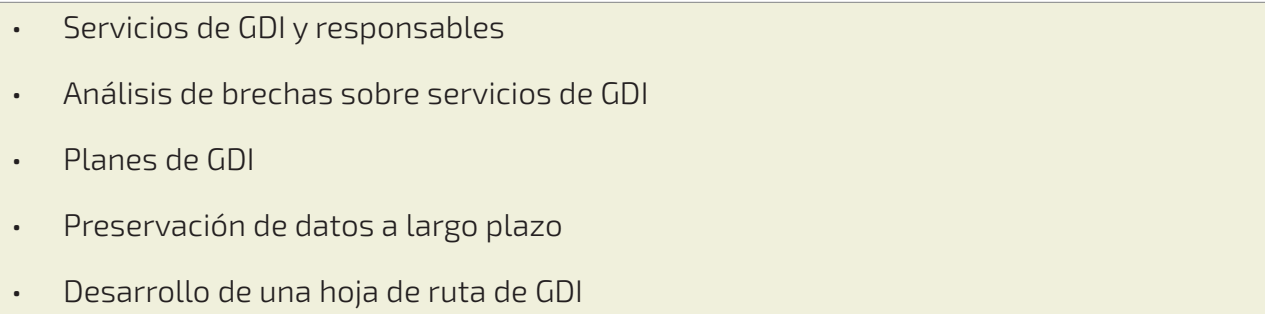 \\
\hline Enlace & https://www.futurelearn.com/courses/delivering-research-data-management-services \\
\hline \multicolumn{2}{|r|}{ Open Science: Sharing your research to the world } \\
\hline Tipo & MOOC - EdX \\
\hline Institución & TU Delft \\
\hline Duración & 4 semanas $(\approx 12 / 15$ horas $)$ \\
\hline Contenido & $\begin{array}{ll}\text { - } & \text { Introducción a la Ciencia Abierta } \\
\text { - } & \text { GDI } \\
\text { - } & \text { Publicar en acceso abierto } \\
\text { - } & \text { Visibilidad de la investigación }\end{array}$ \\
\hline
\end{tabular}




\begin{tabular}{|c|c|}
\hline Enlace & https://www.edx.org/course/open-science-sharing-your-research-with-the-world-2 \\
\hline \multicolumn{2}{|r|}{ Research Data Management and Sharing } \\
\hline Tipo & MOOC - Coursera \\
\hline Institución & Universidad de North Carolina at Chapel Hill y Universidad de Edimburgo \\
\hline Duración & 4 semanas $(\approx 12 / 15$ horas $)$ \\
\hline Contenido & $\begin{array}{l}\text { - Comprensión de los datos de investigación } \\
\text { - Planeamiento de la gestión de datos } \\
\text { - Trabajo con datos } \\
\text { - Compartición de datos }\end{array}$ \\
\hline Enlace & https://www.coursera.org/learn/data-management \\
\hline
\end{tabular}

\section{Anexo 2: Presencia en la web de centros de gestión de datos de investigación o servicios de gestión de datos de investigación}

\begin{tabular}{|c|c|c|c|c|c|c|}
\hline \multicolumn{7}{|c|}{ Web presence of research data management centres or research data management services } \\
\hline \multicolumn{7}{|c|}{ Europe } \\
\hline 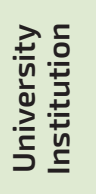 & 趇 & 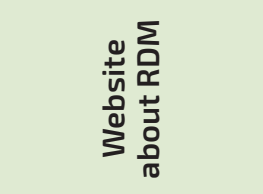 & 冚 & 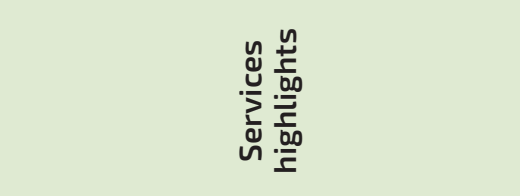 & 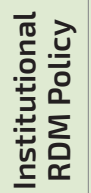 & 㒸 \\
\hline 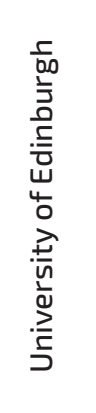 & UK & $\begin{array}{l}\text { https:// } \\
\text { www.ed.ac. } \\
\text { uk/informa- } \\
\text { tion-services/ } \\
\text { research-su- } \\
\text { pport/ } \\
\text { research-da- } \\
\text { ta-service }\end{array}$ & $\begin{array}{l}\text { Information Ser- } \\
\text { vices } \\
\text { » Support for } \\
\text { researchers } \\
\text { »How to publish } \\
\text { and manage your } \\
\text { research } \\
\text { »Research } \\
\text { data service }\end{array}$ & $\begin{array}{l}\text { Pre-project (DMP Tool, per- } \\
\text { sonal consultation - costs } \\
\text { and ethical aspects). Project } \\
\text { (support for finding, accessing } \\
\text { and analysing data, data storage } \\
\text { Electronic Lab Notebook service). } \\
\text { Post-project (data repository). }\end{array}$ & Yes & $\begin{array}{l}\text { https://www. } \\
\text { ed.ac.uk/informa- } \\
\text { tion-services/about/ } \\
\text { policies-and-re- } \\
\text { gulations/ } \\
\text { research-data-policy }\end{array}$ \\
\hline 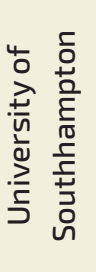 & UK & $\begin{array}{l}\text { http://library. } \\
\text { soton.ac.uk/ } \\
\text { researchdata }\end{array}$ & $\begin{array}{l}\text { Library } \\
\text { » LibGuides@Sou- } \\
\text { thampton } \\
\text { » Research services } \\
\text { »Research data } \\
\text { management }\end{array}$ & $\begin{array}{l}\text { Information about how to create } \\
\text { a DMP, work with data, store and } \\
\text { share them. Trainings about RDM. } \\
\text { Focus on young researchers (PhD). }\end{array}$ & Yes & $\begin{array}{l}\text { http://library.soton. } \\
\text { ac.uk/research- } \\
\text { data/policies }\end{array}$ \\
\hline 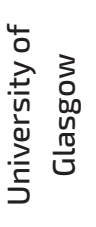 & UK & $\begin{array}{l}\text { https://www. } \\
\text { gla.ac.uk/ } \\
\text { myglasgow/data- } \\
\text { management/ } \\
\text { rdmatglasgow/ }\end{array}$ & $\begin{array}{l}\text { MyGlasgow } \\
\text { »Data management } \\
\text { support for resear- } \\
\text { chers } \\
\text { Team RDM - Library }\end{array}$ & $\begin{array}{l}\text { Information about data storage and } \\
\text { costs, information about working } \\
\text { with data (creation, organisation } \\
\text { and access processes). Focus on } \\
\text { preservation. Trainings about RDM. }\end{array}$ & yes & $\begin{array}{l}\text { https://www. } \\
\text { gla.ac.uk/media/ } \\
\text { Media_555894_ } \\
\text { smxx.pdf }\end{array}$ \\
\hline
\end{tabular}




\begin{tabular}{|c|c|c|c|c|c|c|}
\hline 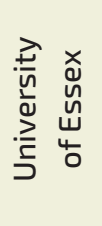 & UK & $\begin{array}{l}\text { https://www1. } \\
\text { essex.ac.uk/reo/ } \\
\text { governance/ } \\
\text { data.aspx }\end{array}$ & $\begin{array}{l}\text { » Research Gover- } \\
\text { nance } \\
\text { » Data (Data Repo- } \\
\text { sitory - library) }\end{array}$ & $\begin{array}{l}\text { Information about DMPs and DMP } \\
\text { creation. Data repository offered } \\
\text { for data sharing. Integration of } \\
\text { Research Development Managers. }\end{array}$ & Yes & $\begin{array}{l}\text { https://www. } \\
\text { essex.ac.uk/staff/ } \\
\text { research-gover- } \\
\text { nance/research-data } \\
\text { (under Policy) }\end{array}$ \\
\hline 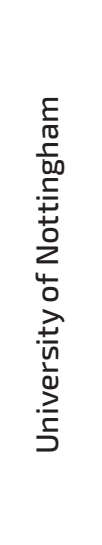 & UK & $\begin{array}{l}\text { https://www. } \\
\text { nottingham. } \\
\text { ac.uk/fabs/rgs/ } \\
\text { research-da- } \\
\text { ta-manage- } \\
\text { ment/index.aspx }\end{array}$ & $\begin{array}{l}\text { Libraries } \\
\text { » Researcher } \\
\text { support } \\
\text { » Research Data } \\
\text { Management }\end{array}$ & $\begin{array}{l}\text { Moodle course about RDM. } \\
\text { Toolbox for DMPs for different } \\
\text { funding agencies. Information } \\
\text { about research data planning. } \\
\text { Information about data archiving } \\
\text { and preservation (Institutional data } \\
\text { repository). Information about data } \\
\text { publishing and sharing. Focus on } \\
\text { ORCID iDs and their internal use } \\
\text { (Nottingham Research Data Mana- } \\
\text { gement Repository, Researchfish } \\
\text { and other university systems). }\end{array}$ & Yes & Closed access \\
\hline 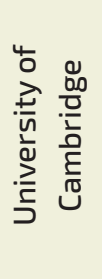 & UK & $\begin{array}{l}\text { https://www. } \\
\text { data.cam.ac.uk/ }\end{array}$ & $\begin{array}{l}\text { University Library } \\
\text { and Research Office }\end{array}$ & $\begin{array}{l}\text { Data Management guides } \\
\text { (create, organise, access data). } \\
\text { Focus on preservation. Data } \\
\text { Repository. Collaboration with } \\
\text { data champions. Training for } \\
\text { library staff and researchers. }\end{array}$ & Yes & $\begin{array}{l}\text { https://www.data. } \\
\text { cam.ac.uk/uni- } \\
\text { versity-policy }\end{array}$ \\
\hline 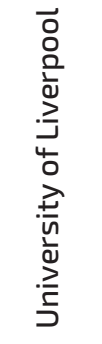 & UK & $\begin{array}{l}\text { https://www. } \\
\text { liverpool. } \\
\text { ac.uk/library/ } \\
\text { research-da- } \\
\text { ta-management/ }\end{array}$ & $\begin{array}{l}\text { Library } \\
\text { » Research data } \\
\text { management }\end{array}$ & $\begin{array}{l}\text { RDM workshops. Guidance about } \\
\text { preparing, managing and sha- } \\
\text { ring data. Focus on FAIR Data } \\
\text { and Open Research. Information } \\
\text { about intellectual property. }\end{array}$ & Yes & $\begin{array}{l}\text { https://www. } \\
\text { liverpool.ac.uk/ } \\
\text { media/livacuk/ } \\
\text { computingservices/ } \\
\text { research-data-management/ } \\
\text { researchdatamana- } \\
\text { gementpolicy.pdf }\end{array}$ \\
\hline 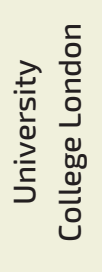 & UK & $\begin{array}{l}\text { https://www.ucl. } \\
\text { ac.uk/library/ } \\
\text { research-su- } \\
\text { pport/ } \\
\text { research-da- } \\
\text { ta-management }\end{array}$ & $\begin{array}{l}\text { Library Service } \\
\text { »Research Support } \\
\text { » Research Data } \\
\text { Management }\end{array}$ & $\begin{array}{l}\text { Learning resources. Guides about } \\
\text { storing, preserving and sharing } \\
\text { data. Guides about handling sen- } \\
\text { sitive and personal information. } \\
\text { Research Data Repository. }\end{array}$ & Yes & $\begin{array}{l}\text { https://www.ucl. } \\
\text { ac.uk/isd/sites/isd/ } \\
\text { files/uclresearchda- } \\
\text { tapolicy_2018.pdf }\end{array}$ \\
\hline 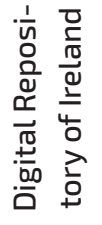 & IE & $\begin{array}{l}\text { https://www.dri. } \\
\text { ie/research-da- } \\
\text { ta-manage- } \\
\text { ment-plans }\end{array}$ & $\begin{array}{l}\text { Research Data } \\
\text { »Research Data } \\
\text { Management Plans }\end{array}$ & $\begin{array}{l}\text { National repository. Core Trust } \\
\text { Seal. Extensive guide about } \\
\text { research data management } \\
\text { plans. Focus on FAIR data. }\end{array}$ & 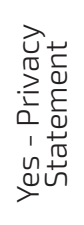 & $\begin{array}{l}\text { https://www.dri.ie/ } \\
\text { dri-privacy-statement }\end{array}$ \\
\hline 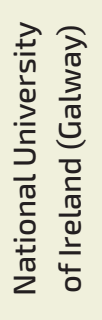 & IE & $\begin{array}{l}\text { https://lib- } \\
\text { guides.library. } \\
\text { nuigalway.ie/ } \\
\text { researchdata- } \\
\text { management/ } \\
\text { ResearchDa- } \\
\text { taWorkingGroup }\end{array}$ & $\begin{array}{l}\text { Library Guides } \\
\text { » Research Data } \\
\text { Management } \\
\text { » Reseach Data } \\
\text { Working Group }\end{array}$ & $\begin{array}{l}\text { Information about: planning data } \\
\text { management, organising and } \\
\text { documenting data, storing and } \\
\text { securing data, preserving and } \\
\text { sharing data. DOI Service (EDIZ). } \\
\text { Guidance about ORCID iD. }\end{array}$ & Yes & $\begin{array}{l}\text { http://www.nui- } \\
\text { galway.ie/media/ } \\
\text { staffsub-sites/ } \\
\text { researchoffice/ } \\
\text { files/Research-Da- } \\
\text { ta-Management-Po- } \\
\text { licy-(QA509).pdf }\end{array}$ \\
\hline
\end{tabular}




\begin{tabular}{|c|c|c|c|c|c|c|}
\hline 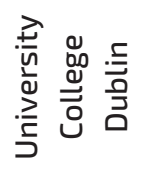 & IE & $\begin{array}{l}\text { https://libgui- } \\
\text { des.ucd.ie/data }\end{array}$ & $\begin{array}{l}\text { Library» LibGuides } \\
\text { » Researcher Guides } \\
\text { » Research Data } \\
\text { Management }\end{array}$ & $\begin{array}{l}\text { Data Manager. DOI registration } \\
\text { service. General support about RDM }\end{array}$ & No & \\
\hline 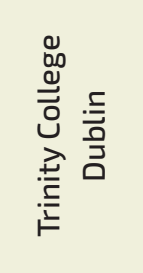 & IE & $\begin{array}{l}\text { https://www. } \\
\text { tcd.ie/library/ } \\
\text { riss/research- } \\
\text { data.php }\end{array}$ & $\begin{array}{l}\text { Library departe- } \\
\text { ment » research } \\
\text { information } \\
\text { system \& services } \\
\text { »research data } \\
\text { management }\end{array}$ & $\begin{array}{l}\text { Support for DMP creation. Informa- } \\
\text { tion about RDM in the lifecycle. }\end{array}$ & No & \\
\hline 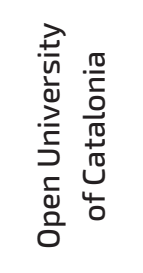 & ES & $\begin{array}{l}\text { http://biblioteca. } \\
\text { uoc.edu/es/ } \\
\text { investigacion/ges- } \\
\text { tion-de-los-da- } \\
\text { tos-de-in- } \\
\text { vestigacion }\end{array}$ & $\begin{array}{l}\text { Library » Investi- } \\
\text { gación Gestión de } \\
\text { la información» } \\
\text { Gestión de los datos } \\
\text { de investigación }\end{array}$ & $\begin{array}{l}\text { Personal support for DMP creation } \\
\text { and repository selection. Informa- } \\
\text { tion about handling and publishing } \\
\text { research data. DMP Tool (DMP } \\
\text { CSUC). Repository (also for research } \\
\text { data) } 02 \text { Dades- Handle PID System. }\end{array}$ & No & \\
\hline 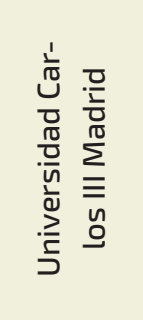 & ES & $\begin{array}{l}\text { https://www. } \\
\text { uc3m.es/ } \\
\text { biblioteca/ } \\
\text { publicacion-difu- } \\
\text { sion-cientifica/ } \\
\text { datos-inves- } \\
\text { tigacion }\end{array}$ & $\begin{array}{l}\text { Library » Carring } \\
\text { out research » } \\
\text { research data }\end{array}$ & $\begin{array}{l}\text { Information about how to cite, } \\
\text { discover, create, process, orga- } \\
\text { nise, share, publish and reuse data. } \\
\text { Personal support for RDM Tasks. } \\
\text { DMP Tool (PGDOnline). Data repo- } \\
\text { sitory, integrated in ecienciaDatos }\end{array}$ & No & \\
\hline 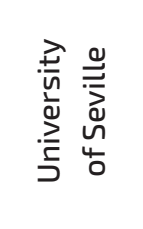 & ES & $\begin{array}{l}\text { https://bib. } \\
\text { us.es/estu- } \\
\text { dia_e_investiga/ } \\
\text { investigacion/ } \\
\text { estrategias/gdi }\end{array}$ & $\begin{array}{l}\text { Investigación » } \\
\text { Estrategias de } \\
\text { publicación » } \\
\text { Gestión de datos } \\
\text { de investigación }\end{array}$ & $\begin{array}{l}\text { Information divided in project } \\
\text { phases. Institutional resposi- } \\
\text { tory adapted for data (idUS) }\end{array}$ & No & \\
\hline 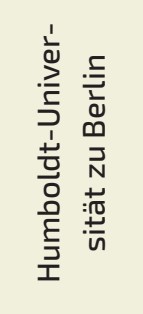 & $\mathrm{DE}$ & $\begin{array}{l}\text { https://www. } \\
\text { cms.hu-berlin. } \\
\text { de/en/dl-en/ } \\
\text { dataman-en/ } \\
\text { welcome?set__ } \\
\text { language=en }\end{array}$ & $\begin{array}{l}\text { »Computer and } \\
\text { media services } \\
\text { (collaboration with } \\
\text { University Library) } \\
\text { »Research Data } \\
\text { Management }\end{array}$ & $\begin{array}{l}\text { Information about working with and } \\
\text { sharing research data. Training and } \\
\text { RDM Material. Different options } \\
\text { for data archiving. Participation in } \\
\text { the project FDMentor (to offer RDM } \\
\text { resources for German speakers). } \\
\text { Integration of Data librarians. }\end{array}$ & Yes & $\begin{array}{l}\text { https://www.cms. } \\
\text { hu-berlin.de/en/ } \\
\text { dl-en/dataman-en/ } \\
\text { info/policy }\end{array}$ \\
\hline 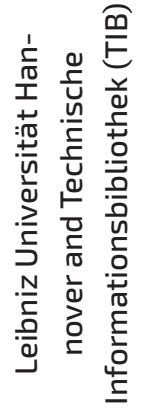 & $\mathrm{DE}$ & $\begin{array}{l}\text { https://www. } \\
\text { fdm.uni-han- } \\
\text { nover.de/en/ }\end{array}$ & Direct & $\begin{array}{l}\text { Guidelines and recommendations. } \\
\text { Training for researchers (focus on } \\
\text { young researchers). DOI Service } \\
\text { (DataCite). Institutional reposi- } \\
\text { tory with DOls. Integration with } \\
\text { ORCID. Data repository RADAR. }\end{array}$ & Yes & $\begin{array}{l}\text { https://www. } \\
\text { uni-hannover.de/ } \\
\text { fileadmin/luh/con- } \\
\text { tent/dezernat4/ } \\
\text { FDM/180417_Guide- } \\
\text { lines_RDM_final.pdf }\end{array}$ \\
\hline 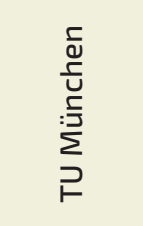 & $\mathrm{DE}$ & $\begin{array}{l}\text { https://www. } \\
\text { ub.tum.de/en/ } \\
\text { eresearch }\end{array}$ & $\begin{array}{l}\text { University Library } \\
\text { » Research data } \\
\text { management }\end{array}$ & $\begin{array}{l}\text { Information about DMPs. Informa- } \\
\text { tion about pushing data. Institutional } \\
\text { repository. Repository mediaTUM. } \\
\text { DOI as PID System. Focus on long- } \\
\text { term archiving and formats. }\end{array}$ & Yes & $\begin{array}{l}\text { https://www. } \\
\text { it.tum.de/projekte/ } \\
\text { forschungsdaten-ma- } \\
\text { nagement/ }\end{array}$ \\
\hline
\end{tabular}




\begin{tabular}{|c|c|c|c|c|c|c|}
\hline 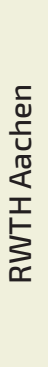 & $\mathrm{DE}$ & $\begin{array}{l}\text { http://www. } \\
\text { rwth-aachen.de/ } \\
\text { cms/root/For- } \\
\text { schung/ Inaw/ } \\
\text { Forschungs- } \\
\text { datenmanage- } \\
\text { ment/lidx/1/ }\end{array}$ & $\begin{array}{l}\text { Research } \\
\text { » Research data } \\
\text { management } \\
\text { (multidiciplinary } \\
\text { team, including } \\
\text { library staff) }\end{array}$ & $\begin{array}{l}\text { Training courses. Information about } \\
\text { archiving and documenting data. } \\
\text { ePIC PID service. Support for DMP } \\
\text { creation -- RDMO and DMPonline. } \\
\text { Tools for collaborative work. }\end{array}$ & Yes & $\begin{array}{l}\text { http://www. } \\
\text { rwth-aachen.de/ } \\
\text { cms/root/Forschung/ } \\
\text { Forschungsdatenma- } \\
\text { nagement/ ncfw/ } \\
\text { Leitlinie-zum-For- } \\
\text { schungsdatenma- } \\
\text { nagement/lidx/1/ }\end{array}$ \\
\hline 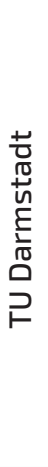 & DE & $\begin{array}{l}\text { https://www. } \\
\text { tu-darmstadt. } \\
\text { de/tudata/ } \\
\text { tudata/digitale_- } \\
\text { forschungsda- } \\
\text { ten_an_der_tu/ } \\
\text { index.de.jsp }\end{array}$ & $\begin{array}{l}\text { Library » TU Data } \\
\text { » Digital } \\
\text { research data }\end{array}$ & $\begin{array}{l}\text { Personal support. Training and } \\
\text { coffee lectures about RDM. Insti- } \\
\text { tutional GitLab. Institutional data } \\
\text { repository (Tudatalin) and DMP } \\
\text { Tool (TUdmo). Multidiciplinary } \\
\text { working group on Research data. }\end{array}$ & Yes & $\begin{array}{l}\text { https://www.tu-dar- } \\
\text { mstadt.de/media/ } \\
\text { daa_responsi- } \\
\text { ves_design/03_for- } \\
\text { schung_medien/ } \\
\text { forschungs- } \\
\text { foerderung_2/ } \\
\text { gute_wiss_praxis/ } \\
\text { Leitlinien_Forschungs- } \\
\text { daten_2015.de.pdf }\end{array}$ \\
\hline 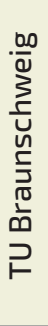 & DE & $\begin{array}{l}\text { https:// } \\
\text { ub.tu-brauns- } \\
\text { chweig.de/ } \\
\text { publizieren_- } \\
\text { openaccess/ } \\
\text { englisch/ } \\
\text { index_en.php }\end{array}$ & $\begin{array}{l}\text { University Library » } \\
\text { Publishing \& Open } \\
\text { Access » Research } \\
\text { Data Management }\end{array}$ & $\begin{array}{l}\text { Preparation of DMPs. Consulta- } \\
\text { tion for archiving and publication } \\
\text { of research data. Legal support. }\end{array}$ & Yes & $\begin{array}{l}\text { https://ub. } \\
\text { tu-braunschweig. } \\
\text { de/publizie- } \\
\text { ren_openaccess/ } \\
\text { forschungsdaten/ } \\
\text { forschungsdaten- } \\
\text { leitlinie.pdf }\end{array}$ \\
\hline 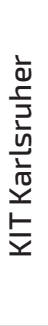 & DE & $\begin{array}{l}\text { https://www. } \\
\text { bibliothek. } \\
\text { kit.edu/cms/ } \\
\text { english/ } \\
\text { research-da- } \\
\text { ta-management. } \\
\text { php }\end{array}$ & $\begin{array}{l}\text { Library » Research } \\
\text { and Publishing } \\
\text { » Research Data } \\
\text { Management }\end{array}$ & $\begin{array}{l}\text { Data repository (KITopen), DOI } \\
\text { assignation and Open Licences. } \\
\text { RDM Team (not in the library). }\end{array}$ & Yes & $\begin{array}{l}\text { http://www.rdm. } \\
\text { kit.edu/downloads/ } \\
\text { KIT-FDM-Policy.pdf }\end{array}$ \\
\hline 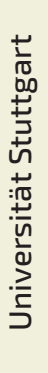 & DE & $\begin{array}{l}\text { https://www. } \\
\text { ub.uni-stuttgart. } \\
\text { de/forschen-pu- } \\
\text { blizieren/ } \\
\text { forschungs- } \\
\text { datenmana- } \\
\text { gement/index. } \\
\text { en.html }\end{array}$ & $\begin{array}{l}\text { Library » Research } \\
\text { \& Publishing } \\
\text { support » Research } \\
\text { Data Management }\end{array}$ & $\begin{array}{l}\text { Information about DMPs, data } \\
\text { storing and data publishing. }\end{array}$ & Yes & $\begin{array}{l}\text { https://www. } \\
\text { ub.uni-stuttgart.de/ } \\
\text { forschen-publizieren/ } \\
\text { FoDa-Policy-English. } \\
\text { pdf }\end{array}$ \\
\hline 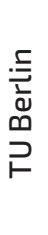 & DE & $\begin{array}{l}\text { https://www. } \\
\text { szf.tu-berlin. } \\
\text { de/menue/ } \\
\text { ueber_das_szf/ } \\
\text { unser_service/ }\end{array}$ & & $\begin{array}{l}\text { Data repository (DepositOnce). } \\
\text { DMP Tool (TUB-DMP). Focus on } \\
\text { FAIR Data. Information according } \\
\text { to the research data life cycle. }\end{array}$ & No & In preparation (2019) \\
\hline 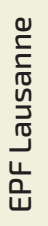 & $\mathrm{CH}$ & $\begin{array}{l}\text { https://www. } \\
\text { epfl.ch/campus/ } \\
\text { library/services/ } \\
\text { services-re- } \\
\text { searchers/ }\end{array}$ & $\begin{array}{l}\text { Library » servi- } \\
\text { ces » services } \\
\text { researchers }\end{array}$ & $\begin{array}{l}\text { DMP Templates, checklist and cost } \\
\text { calculator. Several RDM guides. } \\
\text { Information about file format. } \\
\text { Information about working with, } \\
\text { publishing and preserving data. }\end{array}$ & No & $\begin{array}{l}\text { In preparation https:// } \\
\text { www.dlcm.ch/ser- } \\
\text { vices/dlcm-policy }\end{array}$ \\
\hline
\end{tabular}




\begin{tabular}{|c|c|c|c|c|c|c|}
\hline 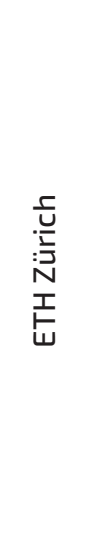 & $\mathrm{CH}$ & $\begin{array}{l}\text { https://www. } \\
\text { ethz.ch/ser- } \\
\text { vices/en/ } \\
\text { service/a-to-z/ } \\
\text { research- } \\
\text { data.html }\end{array}$ & $\begin{array}{l}\text { Services \& Resour- } \\
\text { ces » Research } \\
\text { Data (Library \& } \\
\text { IT Services) }\end{array}$ & $\begin{array}{l}\text { Information about data management } \\
\text { planning, active data management, } \\
\text { publishing and preservation. Par- } \\
\text { ticipation in DLCM (Research Data } \\
\text { Management Hub) Project. Electro- } \\
\text { nic Lab Notebooks and Laboratory } \\
\text { Information Management System » } \\
\text { integrated system openBIS. Ins- } \\
\text { titutional Publications and Data } \\
\text { repository (Research Collection). } \\
\text { DOI National Desk. Trainings and } \\
\text { Workshop on RDM (Library \& IT) }\end{array}$ & No & $\begin{array}{l}\text { Guidelines about } \\
\text { research inte- } \\
\text { grity https://www. } \\
\text { research-collection. } \\
\text { ethz.ch/handle/ } \\
\text { 20.500.11850/179298 }\end{array}$ \\
\hline 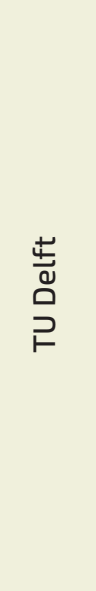 & NL & $\begin{array}{l}\text { https://www. } \\
\text { tudelft.nl/ } \\
\text { en/library/ } \\
\text { current-topics/ } \\
\text { research-da- } \\
\text { ta-management/ }\end{array}$ & $\begin{array}{l}\text { Library » Research } \\
\text { Data Management }\end{array}$ & $\begin{array}{l}\text { Training and workshop. Focus on } \\
\text { young researchers (PhD). Integration } \\
\text { of "The Carpentries" methodology. } \\
\text { Integration of Data Stewards in } \\
\text { each faculty. Information about } \\
\text { DMPs, data discovery and owner- } \\
\text { ship. Data Management costing } \\
\text { tool. Information about experi- } \\
\text { menting with data (data collection, } \\
\text { storing, analysing, visualizing, } \\
\text { and preparation for publication. } \\
\text { Repository } 4 \text { TU.Research Data. } \\
\text { Institutional RDM Policy. }\end{array}$ & Yes & $\begin{array}{l}\text { https://d1rkab7t- } \\
\text { lqy5f1.cloudfront. } \\
\text { net/Library/The- } \\
\text { maportalen/RDM/ } \\
\text { researchdata-fra- } \\
\text { mework-policy.pdf }\end{array}$ \\
\hline 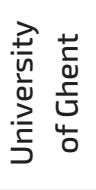 & $\mathrm{BE}$ & $\begin{array}{l}\text { https://www. } \\
\text { ugent.be/en/ } \\
\text { research/data- } \\
\text { management }\end{array}$ & $\begin{array}{l}\text { Research » } \\
\text { Data manage- } \\
\text { ment (Library) }\end{array}$ & $\begin{array}{l}\text { Information about DMPs and } \\
\text { DMP creation (Tool DMP Bel- } \\
\text { gium). Information about } \\
\text { data storing and sharing. }\end{array}$ & Yes & PDF - Home page \\
\hline 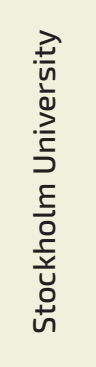 & SE & $\begin{array}{l}\text { https://www. } \\
\text { su.se/english/ } \\
\text { staff/servi- } \\
\text { ces/research/ } \\
\text { research-data/ } \\
\text { data-mana- } \\
\text { gement-servi- } \\
\text { ces-support }\end{array}$ & $\begin{array}{l}\text { Services » Research } \\
\text { » Research Data } \\
\text { Management } \\
\text { (multidisciplinary } \\
\text { team including } \\
\text { library staff) }\end{array}$ & $\begin{array}{l}\text { Information about DMPs and } \\
\text { data sharing. Guidelines for RDM. } \\
\text { Working group on Research Data. } \\
\text { Data repository (Stockholm Uni- } \\
\text { versity Library Dataverse) }\end{array}$ & Yes & $\begin{array}{l}\text { https://www.su.se/ } \\
\text { english/staff/organi- } \\
\text { sation-governance/ } \\
\text { governing-docu- } \\
\text { ments-rules-and-re- } \\
\text { gulations/research/ } \\
\text { research-data-po- } \\
\text { licy-1.387809 }\end{array}$ \\
\hline 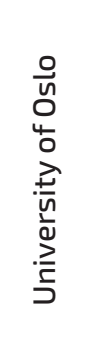 & NO & $\begin{array}{l}\text { https://www. } \\
\text { uio.no/english/ } \\
\text { for-emplo- } \\
\text { yees/support/ } \\
\text { research/ } \\
\text { research-da- } \\
\text { ta-management/ }\end{array}$ & $\begin{array}{l}\text { For employees » } \\
\text { Research Support } \\
\text { » Research Data } \\
\text { Management } \\
\text { (multidisciplinary } \\
\text { team including } \\
\text { library staff) }\end{array}$ & $\begin{array}{l}\text { Trainings and workshops (the } \\
\text { Carpentries Methodology). } \\
\text { Information about DMPs (diffe- } \\
\text { rent tools including a national } \\
\text { one NSD). Information about } \\
\text { data archiving (national } \\
\text { archive). Focus on FAIR Data }\end{array}$ & Yes & $\begin{array}{l}\text { https://www. } \\
\text { uio.no/english/ } \\
\text { for-employees/ } \\
\text { support/research/ } \\
\text { research-da- } \\
\text { ta-management/ } \\
\text { policies-and-guide- } \\
\text { lines/index.html }\end{array}$ \\
\hline 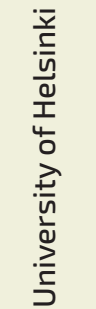 & $\mathrm{FI}$ & $\begin{array}{l}\text { https://www. } \\
\text { helsinki.fi/ } \\
\text { en/research/ } \\
\text { research-en- } \\
\text { vironment/ } \\
\text { research-da- } \\
\text { ta-management }\end{array}$ & $\begin{array}{l}\text { Research --> } \\
\text { Research Environ- } \\
\text { ment --> Research } \\
\text { Data Management } \\
\text { (multidisciplinary } \\
\text { team including } \\
\text { library staff) }\end{array}$ & $\begin{array}{l}\text { Information about data manage- } \\
\text { ment planning. National DMP Tool } \\
\text { (DMP Tuuli). Information about } \\
\text { ethical and legal aspect. Data } \\
\text { Documentation. Information about } \\
\text { storing data (internal systems) and } \\
\text { sharing data (external repositories). }\end{array}$ & Yes & $\begin{array}{l}\text { https://www.helsinki. } \\
\text { fi/en/research/ } \\
\text { research-environ- } \\
\text { ment/research-data/ } \\
\text { research-data-policy }\end{array}$ \\
\hline
\end{tabular}




\begin{tabular}{|c|c|c|c|c|c|c|}
\hline 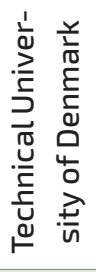 & DK & $\begin{array}{l}\text { https://www. } \\
\text { dtu.dk/english/ } \\
\text { research/ } \\
\text { research-at-dtu/ } \\
\text { research-da- } \\
\text { ta-management }\end{array}$ & $\begin{array}{l}\text { Resarch --> } \\
\text { Research at DTU } \\
\text {--> Research Data } \\
\text { Management } \\
\text { (Contact @Library) }\end{array}$ & $\begin{array}{l}\text { Information about DMPs, data } \\
\text { collection, storing and data } \\
\text { publishing. Institutional data } \\
\text { repository (DTU Data - Figs- } \\
\text { hare). DOI assignation. }\end{array}$ & Yes & PDF - Home page \\
\hline \multicolumn{7}{|c|}{ Latin America } \\
\hline 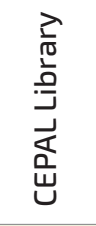 & & $\begin{array}{l}\text { https://biblio- } \\
\text { guias.cepal.org/ } \\
\text { gestion-de-da- } \\
\text { tos-de-in- } \\
\text { vestigacion }\end{array}$ & $\begin{array}{l}\text { Biblioguias » } \\
\text { Gestión de datos } \\
\text { de investigación }\end{array}$ & $\begin{array}{l}\text { Very complete guide on RDM. } \\
\text { Collaboration with LEARN pro- } \\
\text { ject. Webinars about RDM } \\
\text { and Data Stewards in coo- } \\
\text { peration with TU Delft. }\end{array}$ & no & \\
\hline 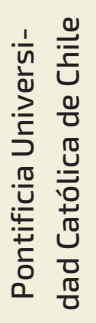 & $C L$ & $\begin{array}{l}\text { https://guias- } \\
\text { tematicas. } \\
\text { bibliotecas. } \\
\text { uc.cl/gdi_ip }\end{array}$ & $\begin{array}{l}\text { Biblioteca » Guías } \\
\text { temáticas » Ges- } \\
\text { tión de datos de } \\
\text { investigación }\end{array}$ & $\begin{array}{l}\text { Information about RDM. Informa- } \\
\text { tion about DMPs (selection of tools). } \\
\text { Information about data formats, } \\
\text { data selection and naming. Infor- } \\
\text { mation about ethical and legal } \\
\text { aspects. Implementation of ORCID } \\
\text { in the institutional repository. }\end{array}$ & No & $\begin{array}{l}\text { RDM Policy proposal } \\
\text { CONICYT http://datos- } \\
\text { cientificos.cl/politica }\end{array}$ \\
\hline
\end{tabular}

El Observatorio de Cibermedios es una producción del Grupo de Investigación en Documentación Digital y Comunicación Interactiva (DigiDoc) del Departamento de Comunicación de la Universitat Pompeu Fabra.

El Observatorio de Cibermedios (OCM) forma parte del proyecto del Plan Nacional "Creación y contenido interactivo en la comunicación de información audiovisual: audiencias, diseño, sistemas y formatos". CS02015-64955-C4-2-R (MINECO/ FEDER), Ministerio de Economía y Competitividad (España). 